\title{
Reactions of $d^{0}$ Group 4 Amides with Dioxygen. Preparation of Unusual Oxo Aminoxy Complexes and Theoretical Studies of Their Formation
}

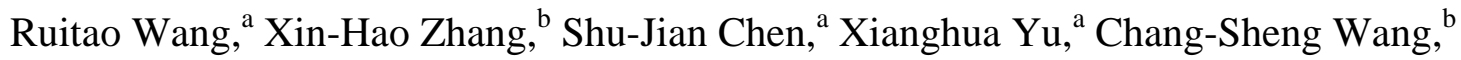 \\ David B. Beach, ${ }^{\mathrm{c}}$ Yun-Dong Wu, ${ }^{\mathrm{b}, *}$ and Zi-Ling Xue ${ }^{\mathrm{a}, *}$ \\ ${ }^{a}$ Department of Chemistry, The University of Tennessee, Knoxville, Tennessee 37996- \\ 1600 \\ ${ }^{\mathrm{b}}$ Department of Chemistry, The Hong Kong University of Science and Technology, Clear \\ Water Bay, Kowloon, Hong Kong, China \\ c Chemical Sciences Division, Oak Ridge National Laboratory, Oak Ridge, Tennessee 37831- \\ 6197
}

\section{Supporting Information}




\section{References with more than 10 authors.}

(1c) Kelly, P. V.; Mooney, M. B.; Beechinor, J. T.; O’Sullivan, B. J.; Hurley, P. K.; Crean, G. M.; Zhang, J.-Y.; Boyd, I. W.; Paillous, M.; Jimenez, C.; Senateur, J.-P. Adv. Mater. Opt. Electron. 2000, 10, 115.

(30b) Liu, X.-Z.; Wu, Z.-Z.; Cai, H.; Yang, Y.-H.; Chen, T.-N.; Vallet, C. E.; Zuhr, R. A.;

Beach, D. B.; Peng, Z.-H.; Wu, Y.-D.; Concolino, T. E.; Rheingold, A. L.; Xue, Z.-L. J. Am. Chem. Soc. 2001, 123, 8011.

(37) Gaussian 03, Revision B.03. Frisch, M. J.; Trucks, G. W.; Schlegel, H. B.; Scuseria, G. E.;

Robb, M. A.; Cheeseman, J. R.; Montgomery, J. A.; Vreven, Jr., T.; Kudin, K. N.; Burant, J. C.; Millam, J. M.; Iyengar, S. S.; Tomasi, J.; Barone, V.; Mennucci, B.; Cossi, M.; Scalmani, G.; Rega, N.; Petersson, G. A.; Nakatsuji, H.; Hada, M.; Ehara, M.; Toyota, K.; Fukuda, R.; Hasegawa, J.; Ishida, M.; Nakajima, T.; Honda, Y.; Kitao, O.; Nakai, H.; Klene, M.; Li, X.; Knox, J. E.; Hratchian, H. P.; Cross, J. B.; Adamo, C.; Jaramillo, J.; Gomperts, R.; Stratmann, R. E.; Yazyev, O.; Austin, A. J.; Cammi, R.; Pomelli, C.; Ochterski, J. W.; Ayala, P. Y.; Morokuma, K.; Voth, G. A.; Salvador, P.; Dannenberg, J. J.; Zakrzewski, V. G.; Dapprich, S.; Daniels, A. D.;

Strain, M. C.; Farkas, O.; Malick, D. K.; Rabuck, A. D.; Raghavachari, K.; Foresman, J. B.; Ortiz, J. V.; Cui, Q.; Baboul, A. G.; Clifford, S.; Cioslowski, J.; Stefanov, B. B.; Liu, G.; Liashenko, A.; Piskorz, P.; Komaromi, I.; Martin, R. L.; Fox, D. J.; Keith, T.; Al-Laham, M. A.; Peng, C. Y.; Nanayakkara, A.; Challacombe, M.; Gill, P. M. W.; Johnson, B.; Chen, W.; Wong, M. W.; Gonzalez, C.; Pople, J. A. Gaussian, Inc., Pittsburgh, PA, 2003. 


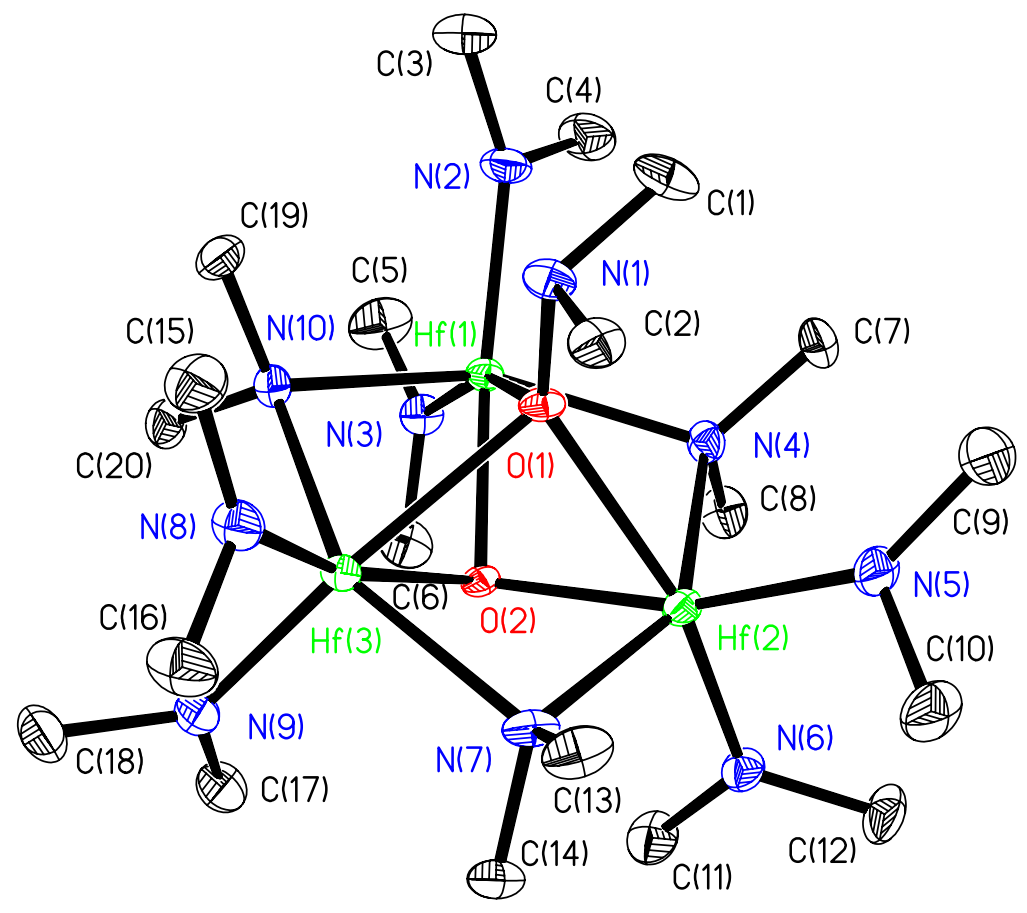


Figure S1. ORTEP of $\left(\mathrm{Me}_{2} \mathrm{~N}\right)_{6} \mathrm{Hf}_{3}\left(\mu-\mathrm{NMe}_{2}\right)_{3}\left(\mu_{3}-\mathrm{O}\right)\left(\mu_{3}-\mathrm{ONMe}_{2}\right)(4)$ showing $30 \%$ probability thermal ellipsoids. The $\mathrm{H}$ atoms have been omitted for clarity. The structure of this complex is isomorphous to that of its $\mathrm{Zr}$ analog 3 (Figure 1). 


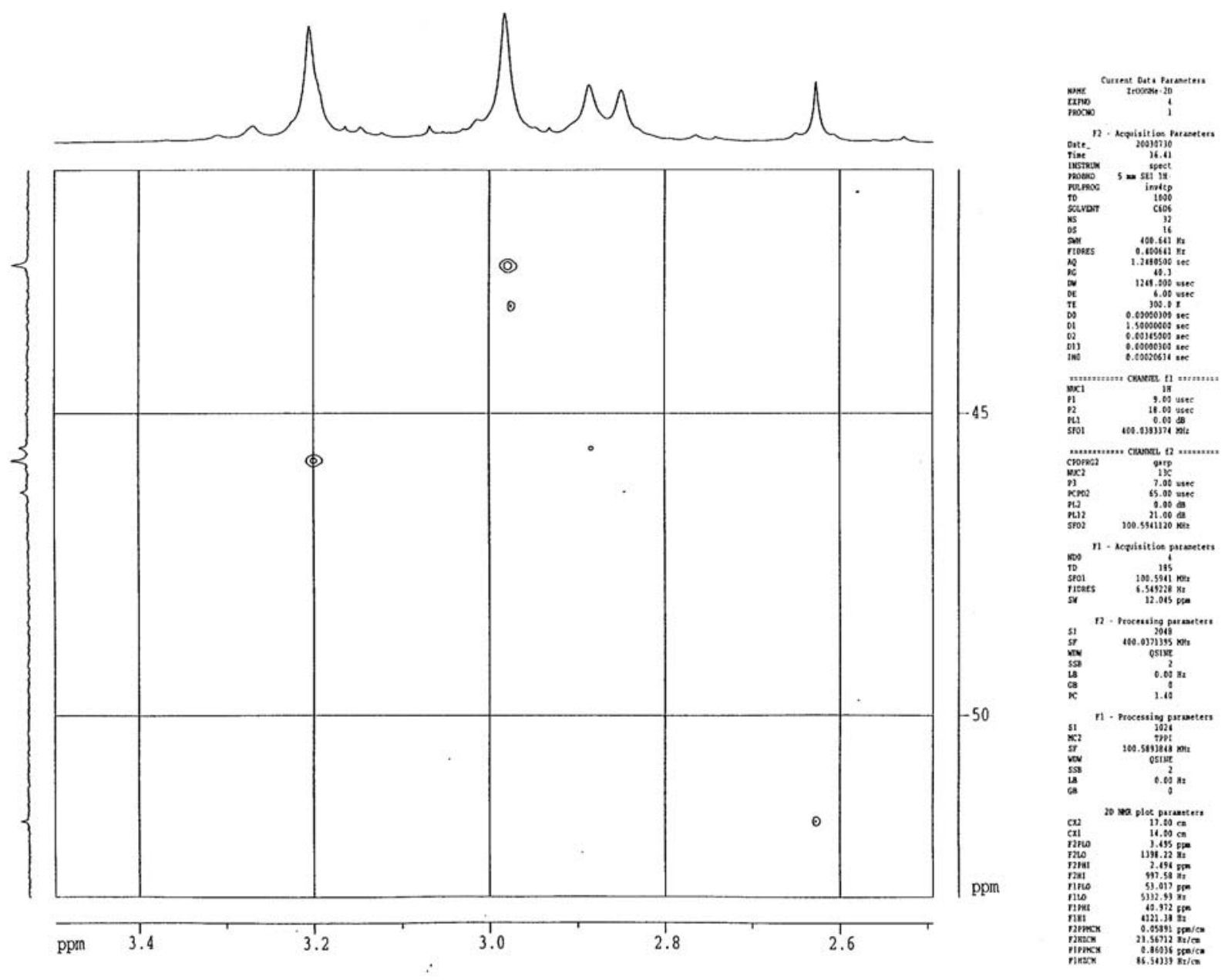

Figure S2a. HMQC NMR spectrum of $\mathrm{Zr}_{3}\left(\mathrm{NMe}_{2}\right)_{6}\left(\mu-\mathrm{NMe}_{2}\right)_{3}\left(\mu_{3}-\mathrm{O}\right)\left(\mu_{3}-\mathrm{ONMe}_{2}\right)(3)$ at $23{ }^{\circ} \mathrm{C}$. 


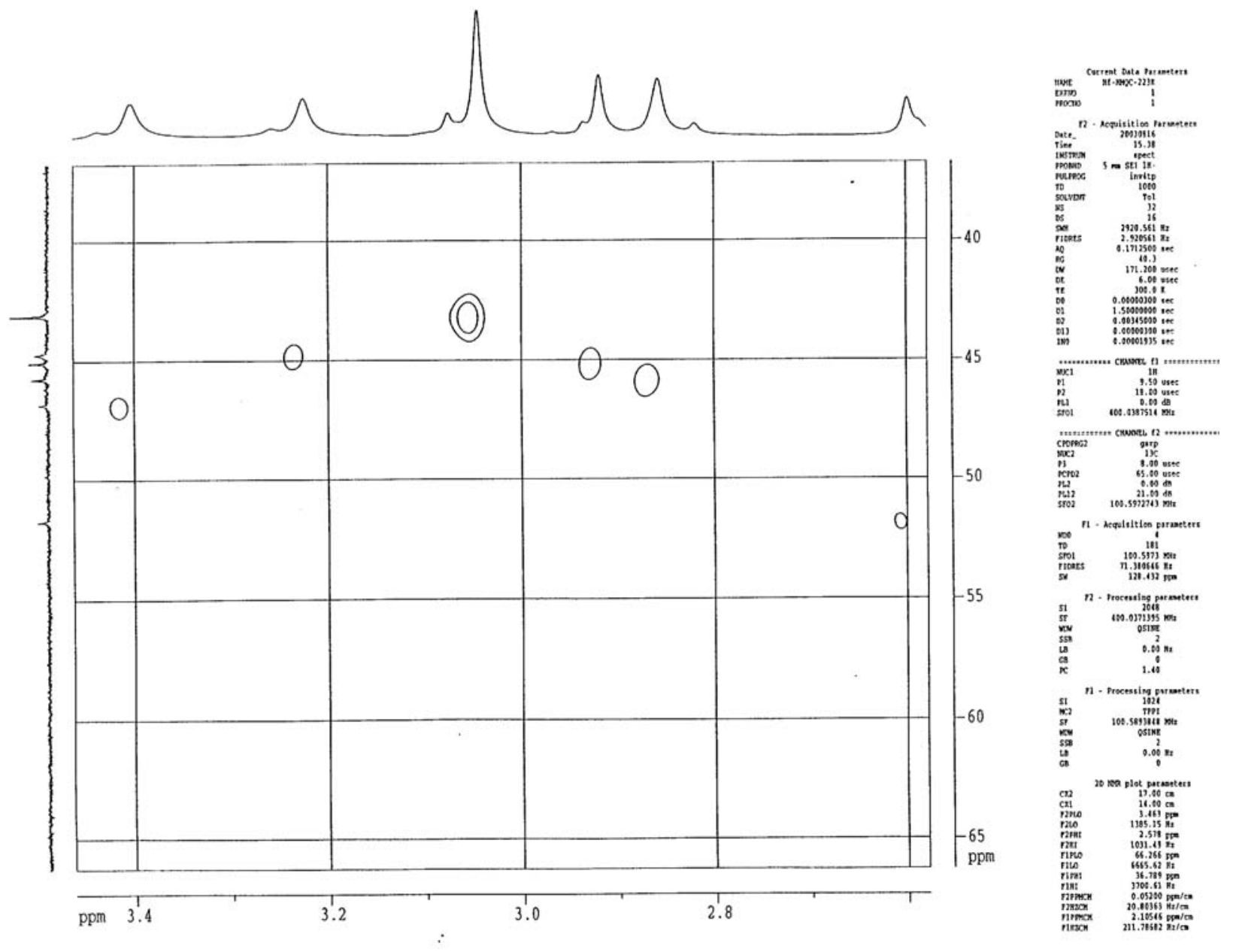

Figure S2b. HMQC NMR spectrum of $\mathrm{Hf}_{3}\left(\mathrm{NMe}_{2}\right)_{6}\left(\mu-\mathrm{NMe}_{2}\right)_{3}\left(\mu_{3}-\mathrm{O}\right)\left(\mu_{3}-\mathrm{ONMe}_{2}\right)(4)$ at $-50{ }^{\circ} \mathrm{C}$. 
Table S1. Crystal data and structure refinement for 3.

\begin{tabular}{|c|c|c|}
\hline Empirical formula & \multicolumn{2}{|l|}{$\mathrm{C}_{20} \mathrm{H}_{60} \mathrm{~N}_{10} \mathrm{O}_{2} \mathrm{Zr}_{3}$} \\
\hline Formula weight & \multicolumn{2}{|l|}{746.44} \\
\hline Temperature & \multicolumn{2}{|l|}{$-100(2)^{\circ} \mathrm{C}$} \\
\hline Wavelength & \multicolumn{2}{|l|}{$0.71073 \AA$} \\
\hline Crystal system & \multicolumn{2}{|l|}{ Orthorhombic } \\
\hline Space group & \multicolumn{2}{|l|}{ Fdd2 } \\
\hline \multirow[t]{3}{*}{ Unit cell dimensions } & $\mathrm{a}=25.767(10) \AA$ & $\alpha=90^{\circ}$ \\
\hline & $\mathrm{b}=28.493(17) \AA$ & $\beta=90^{\circ}$ \\
\hline & $c=19.631(7) \AA$ & $\gamma=90^{\circ}$ \\
\hline Volume & $14413(12) \AA^{3}$ & \\
\hline $\mathrm{Z}$ & 16 & \\
\hline Density (calculated) & $1.376 \mathrm{~g} / \mathrm{cm}^{3}$ & \\
\hline Absorption coefficient & $0.881 \mathrm{~mm}^{-1}$ & \\
\hline $\mathrm{F}(000)$ & 6176 & \\
\hline Crystal size & $0.65 \times 0.53 \times 0.45$ & \\
\hline Theta range for data collection & 1.49 to $28.36^{\circ}$ & \\
\hline Index ranges & $-33 \leq \mathrm{h} \leq 34,-37 \leq$ & , $-25 \leq 1 \leq 25$ \\
\hline Reflections collected & 33464 & \\
\hline Independent reflections & $8579[\mathrm{R}(\mathrm{int})=0.0$ & \\
\hline Completeness to theta $=28.36^{\circ}$ & $97.2 \%$ & \\
\hline Absorption correction & Semi-empirical frc & ivalents \\
\hline Max. and min. transmission & 0.6925 and 0.5981 & \\
\hline Refinement method & Full-matrix least-s & on $\mathrm{F}^{2}$ \\
\hline Data / restraints / parameters & 8579 / 1 / 336 & \\
\hline Goodness-of-fit on $\mathrm{F}^{2}$ & 0.855 & \\
\hline Final R indices $[\mathrm{I}>2 \operatorname{sigma}(\mathrm{I})]$ & $\mathrm{R} 1=0.0275, \mathrm{wR} 2$ & \\
\hline $\mathrm{R}$ indices (all data) & $\mathrm{R} 1=0.0292, \mathrm{wR} 2$ & \\
\hline Absolute structure parameter & $0.99(4)$ & \\
\hline Largest diff. peak and hole & 0.909 and $-0.353 \epsilon$ & \\
\hline
\end{tabular}


Table S2. Atomic coordinates $\left(\times 10^{4}\right)$ and equivalent isotropic displacement parameters $\left(\AA^{2} \times\right.$ $10^{3}$ ) for 3 . $U(e q)$ is defined as one third of the trace of the orthogonalized $\mathrm{Uij}^{\mathrm{ij}}$ tensor.

\begin{tabular}{|c|c|c|c|c|}
\hline & $\mathrm{X}$ & $\mathrm{y}$ & $\mathrm{Z}$ & $\mathrm{U}(\mathrm{eq})$ \\
\hline $\mathrm{C}(1)$ & 2772(2) & $9620(2)$ & $-1086(2)$ & $48(1)$ \\
\hline $\mathrm{C}(2)$ & $2328(2)$ & 8926(1) & $-775(2)$ & $40(1)$ \\
\hline C(3) & $2565(2)$ & $10736(2)$ & $1994(2)$ & $49(1)$ \\
\hline C(4) & $3406(2)$ & 10899(2) & 1545(3) & $58(1)$ \\
\hline C(5) & $3836(2)$ & 10098(2) & $-352(3)$ & $66(1)$ \\
\hline C(6) & $3434(2)$ & 10819(2) & $-129(3)$ & $60(1)$ \\
\hline C(7) & $3263(2)$ & 9621(1) & $1878(2)$ & $41(1)$ \\
\hline C(8) & $3506(1)$ & $9272(1)$ & 830(2) & $42(1)$ \\
\hline C(9) & 1992(2) & $9477(2)$ & 2594(2) & $47(1)$ \\
\hline $\mathrm{C}(10)$ & 2391(2) & $8731(2)$ & $2574(2)$ & $55(1)$ \\
\hline $\mathrm{C}(11)$ & $2162(2)$ & $8156(2)$ & 1141(3) & $55(1)$ \\
\hline $\mathrm{C}(12)$ & 2895(2) & $8255(1)$ & 429(2) & $49(1)$ \\
\hline C(13) & 1089(1) & 9197(1) & 1311(2) & $39(1)$ \\
\hline $\mathrm{C}(14)$ & $1247(2)$ & 8791(2) & 290(2) & $49(1)$ \\
\hline C(15) & $967(2)$ & $10389(2)$ & $1413(2)$ & $49(1)$ \\
\hline $\mathrm{C}(16)$ & $549(2)$ & $10509(2)$ & 353(2) & $58(1)$ \\
\hline $\mathrm{C}(17)$ & 769(2) & 9614(3) & $-706(3)$ & $70(2)$ \\
\hline C(18) & $1386(2)$ & 9922(3) & $-1458(3)$ & $91(2)$ \\
\hline C(19) & $1914(2)$ & 10960(1) & 464(2) & $46(1)$ \\
\hline $\mathrm{C}(20)$ & 2184(2) & 10719(2) & $-634(2)$ & $49(1)$ \\
\hline $\mathrm{N}(1)$ & 2679(1) & 9287(1) & $-529(1)$ & $33(1)$ \\
\hline $\mathrm{N}(2)$ & 2920(1) & 10649(1) & $1428(2)$ & $37(1)$ \\
\hline $\mathrm{N}(3)$ & $3413(1)$ & 10320(1) & 20(2) & $41(1)$ \\
\hline $\mathrm{N}(4)$ & $3090(1)$ & 9528(1) & 1178(1) & $29(1)$ \\
\hline $\mathrm{N}(5)$ & 2194(1) & 9111(1) & $2156(2)$ & $37(1)$ \\
\hline $\mathrm{N}(6)$ & $2514(1)$ & 8501(1) & $843(2)$ & $38(1)$ \\
\hline $\mathrm{N}(7)$ & $1440(1)$ & 9175(1) & 722(1) & $33(1)$ \\
\hline $\mathrm{N}(8)$ & 975(1) & 10270(1) & 692(2) & $37(1)$ \\
\hline N(9) & $1277(2)$ & 9843(2) & $-749(2)$ & $54(1)$ \\
\hline $\mathrm{N}(10)$ & 2108(1) & 10552(1) & 67(1) & $33(1)$ \\
\hline $\mathrm{O}(1)$ & 2094(1) & 9895(1) & 1065(1) & $24(1)$ \\
\hline $\mathrm{O}(2)$ & $2409(1)$ & 9538(1) & 21(1) & $26(1)$ \\
\hline $\operatorname{Zr}(1)$ & 2778(1) & 10188(1) & $643(1)$ & $25(1)$ \\
\hline $\operatorname{Zr}(2)$ & $1570(1)$ & 9906(1) & 234(1) & $27(1)$ \\
\hline $\operatorname{Zr}(3)$ & 2292(1) & $9175(1)$ & 1119(1) & $26(1)$ \\
\hline
\end{tabular}


Table S3. Bond lengths $(\AA)$ and angles $\left({ }^{\circ}\right)$ for 3.

\begin{tabular}{|c|c|c|c|}
\hline $\mathrm{C}(1)-\mathrm{N}(1)$ & $1.467(5)$ & $\mathrm{N}(2)-\operatorname{Zr}(1)$ & $2.057(3)$ \\
\hline $\mathrm{C}(2)-\mathrm{N}(1)$ & $1.452(5)$ & $\mathrm{N}(3)-\operatorname{Zr}(1)$ & $2.078(3)$ \\
\hline $\mathrm{C}(3)-\mathrm{N}(2)$ & $1.460(5)$ & $N(4)-Z r(3)$ & $2.291(3)$ \\
\hline $\mathrm{C}(4)-\mathrm{N}(2)$ & $1.459(5)$ & $\mathrm{N}(4)-\operatorname{Zr}(1)$ & $2.300(3)$ \\
\hline $\mathrm{C}(5)-\mathrm{N}(3)$ & $1.457(6)$ & $N(5)-Z r(3)$ & $2.059(3)$ \\
\hline $\mathrm{C}(6)-\mathrm{N}(3)$ & $1.451(6)$ & $N(6)-\operatorname{Zr}(3)$ & $2.075(3)$ \\
\hline $\mathrm{C}(7)-\mathrm{N}(4)$ & $1.469(4)$ & $N(7)-\operatorname{Zr}(2)$ & $2.316(3)$ \\
\hline $\mathrm{C}(8)-\mathrm{N}(4)$ & $1.465(4)$ & $\mathrm{N}(7)-\operatorname{Zr}(3)$ & 2.331(3) \\
\hline $\mathrm{C}(9)-\mathrm{N}(5)$ & $1.448(5)$ & $\mathrm{N}(8)-\operatorname{Zr}(2)$ & $2.058(3)$ \\
\hline C(10)-N(5) & $1.450(5)$ & $\mathrm{N}(9)-\operatorname{Zr}(2)$ & $2.080(4)$ \\
\hline $\mathrm{C}(11)-\mathrm{N}(6)$ & $1.462(5)$ & $\mathrm{N}(10)-\operatorname{Zr}(1)$ & $2.308(3)$ \\
\hline $\mathrm{C}(12)-\mathrm{N}(6)$ & $1.455(5)$ & $\mathrm{N}(10)-\operatorname{Zr}(2)$ & $2.328(3)$ \\
\hline $\mathrm{C}(13)-\mathrm{N}(7)$ & $1.469(4)$ & $\mathrm{O}(1)-\mathrm{Zr}(3)$ & $2.116(2)$ \\
\hline $\mathrm{C}(14)-\mathrm{N}(7)$ & $1.471(4)$ & $\mathrm{O}(1)-\mathrm{Zr}(1)$ & $2.119(2)$ \\
\hline $\mathrm{C}(15)-\mathrm{N}(8)$ & $1.457(5)$ & $\mathrm{O}(1)-\mathrm{Zr}(2)$ & $2.119(2)$ \\
\hline $\mathrm{C}(16)-\mathrm{N}(8)$ & $1.454(5)$ & $\mathrm{O}(2)-\mathrm{Zr}(3)$ & $2.409(2)$ \\
\hline $\mathrm{C}(17)-\mathrm{N}(9)$ & $1.465(7)$ & $\mathrm{O}(2)-\mathrm{Zr}(1)$ & $2.415(2)$ \\
\hline C(18)-N(9) & $1.437(6)$ & $\mathrm{O}(2)-\mathrm{Zr}(2)$ & $2.440(2)$ \\
\hline $\mathrm{C}(19)-\mathrm{N}(10)$ & $1.486(4)$ & $\operatorname{Zr}(1)-\operatorname{Zr}(3)$ & $3.2825(16)$ \\
\hline $\mathrm{C}(20)-\mathrm{N}(10)$ & $1.470(5)$ & $\operatorname{Zr}(1)-\operatorname{Zr}(2)$ & $3.3149(12)$ \\
\hline $\mathrm{N}(1)-\mathrm{O}(2)$ & $1.469(3)$ & $\operatorname{Zr}(2)-\operatorname{Zr}(3)$ & $3.2896(10)$ \\
\hline $\mathrm{C}(1)-\mathrm{N}(1)-\mathrm{C}(2)$ & $108.1(3)$ & $\mathrm{C}(14)-\mathrm{N}(7)-\operatorname{Zr}(2)$ & $118.7(3)$ \\
\hline $\mathrm{C}(1)-\mathrm{N}(1)-\mathrm{O}(2)$ & 108.1(3) & $\mathrm{C}(13)-\mathrm{N}(7)-\operatorname{Zr}(3)$ & $108.4(2)$ \\
\hline $\mathrm{C}(2)-\mathrm{N}(1)-\mathrm{O}(2)$ & $107.2(2)$ & $\mathrm{C}(14)-\mathrm{N}(7)-\operatorname{Zr}(3)$ & $120.7(2)$ \\
\hline $\mathrm{C}(4)-\mathrm{N}(2)-\mathrm{C}(3)$ & 109.6(3) & $\operatorname{Zr}(2)-N(7)-Z r(3)$ & 90.12(9) \\
\hline $\mathrm{C}(4)-\mathrm{N}(2)-\mathrm{Zr}(1)$ & $125.6(3)$ & $\mathrm{C}(16)-\mathrm{N}(8)-\mathrm{C}(15)$ & $108.9(3)$ \\
\hline $\mathrm{C}(3)-\mathrm{N}(2)-\mathrm{Zr}(1)$ & $124.5(2)$ & $\mathrm{C}(16)-\mathrm{N}(8)-\operatorname{Zr}(2)$ & $126.8(3)$ \\
\hline $\mathrm{C}(6)-\mathrm{N}(3)-\mathrm{C}(5)$ & $107.3(4)$ & $\mathrm{C}(15)-\mathrm{N}(8)-\operatorname{Zr}(2)$ & $123.6(2)$ \\
\hline C(6)-N(3)-Zr(1) & $109.0(3)$ & $\mathrm{C}(18)-\mathrm{N}(9)-\mathrm{C}(17)$ & $107.6(4)$ \\
\hline $\mathrm{C}(5)-\mathrm{N}(3)-\mathrm{Zr}(1)$ & 143.7(3) & $\mathrm{C}(18)-\mathrm{N}(9)-\operatorname{Zr}(2)$ & $144.4(4)$ \\
\hline $\mathrm{C}(8)-\mathrm{N}(4)-\mathrm{C}(7)$ & 107.7(3) & $\mathrm{C}(17)-\mathrm{N}(9)-\mathrm{Zr}(2)$ & 107.9(3) \\
\hline $\mathrm{C}(8)-\mathrm{N}(4)-\mathrm{Zr}(3)$ & $114.5(2)$ & $\mathrm{C}(20)-\mathrm{N}(10)-\mathrm{C}(19)$ & 106.3(3) \\
\hline $\mathrm{C}(7)-\mathrm{N}(4)-\mathrm{Zr}(3)$ & $113.5(2)$ & $\mathrm{C}(20)-\mathrm{N}(10)-\mathrm{Zr}(1)$ & $120.3(2)$ \\
\hline C(8)-N(4)-Zr(1) & $116.7(2)$ & $\mathrm{C}(19)-\mathrm{N}(10)-\mathrm{Zr}(1)$ & $110.2(2)$ \\
\hline $\mathrm{C}(7)-\mathrm{N}(4)-\mathrm{Zr}(1)$ & $112.7(2)$ & $\mathrm{C}(20)-\mathrm{N}(10)-\mathrm{Zr}(2)$ & $117.8(2)$ \\
\hline $\mathrm{Zr}(3)-\mathrm{N}(4)-\mathrm{Zr}(1)$ & 91.28(9) & $\mathrm{C}(19)-\mathrm{N}(10)-\mathrm{Zr}(2)$ & $110.2(2)$ \\
\hline $\mathrm{C}(9)-\mathrm{N}(5)-\mathrm{C}(10)$ & 109.1(3) & $\operatorname{Zr}(1)-N(10)-\operatorname{Zr}(2)$ & 91.28(10) \\
\hline C(9)-N(5)-Zr(3) & $124.6(2)$ & $\mathrm{Zr}(3)-\mathrm{O}(1)-\mathrm{Zr}(1)$ & 101.62(9) \\
\hline $\mathrm{C}(10)-\mathrm{N}(5)-\operatorname{Zr}(3)$ & 125.6(3) & $\mathrm{Zr}(3)-\mathrm{O}(1)-\operatorname{Zr}(2)$ & 101.94(8) \\
\hline $\mathrm{C}(11)-\mathrm{N}(6)-\mathrm{C}(12)$ & 108.6(3) & $\operatorname{Zr}(1)-\mathrm{O}(1)-\operatorname{Zr}(2)$ & 102.92(9) \\
\hline $\mathrm{C}(11)-\mathrm{N}(6)-\mathrm{Zr}(3)$ & $110.3(2)$ & $\mathrm{N}(1)-\mathrm{O}(2)-\mathrm{Zr}(3)$ & $120.51(17)$ \\
\hline $\mathrm{C}(12)-\mathrm{N}(6)-\operatorname{Zr}(3)$ & 141.1(3) & $\mathrm{N}(1)-\mathrm{O}(2)-\mathrm{Zr}(1)$ & 123.99(16) \\
\hline $\mathrm{C}(13)-\mathrm{N}(7)-\mathrm{C}(14)$ & 106.2(3) & $\mathrm{Zr}(3)-\mathrm{O}(2)-\mathrm{Zr}(1)$ & $85.76(7)$ \\
\hline $\mathrm{C}(13)-\mathrm{N}(7)-\mathrm{Zr}(2)$ & $112.1(2)$ & $\mathrm{N}(1)-\mathrm{O}(2)-\mathrm{Zr}(2)$ & 138.93(17) \\
\hline
\end{tabular}




\begin{tabular}{|c|c|c|c|}
\hline $\operatorname{Zr}(3)-\mathrm{O}(2)-\operatorname{Zr}(2)$ & $85.44(6)$ & $\mathrm{N}(10)-\mathrm{Zr}(2)-\mathrm{O}(2)$ & 77.72(9) \\
\hline $\operatorname{Zr}(1)-O(2)-\operatorname{Zr}(2)$ & 86.13(7) & $\mathrm{N}(8)-\operatorname{Zr}(2)-\operatorname{Zr}(3)$ & 120.66(9) \\
\hline $\mathrm{N}(2)-\mathrm{Zr}(1)-\mathrm{N}(3)$ & 100.70(13) & $\mathrm{N}(9)-\operatorname{Zr}(2)-\operatorname{Zr}(3)$ & 129.93(13) \\
\hline $\mathrm{N}(2)-\mathrm{Zr}(1)-\mathrm{O}(1)$ & $96.18(10)$ & $\mathrm{O}(1)-\operatorname{Zr}(2)-\operatorname{Zr}(3)$ & $39.00(6)$ \\
\hline $\mathrm{N}(3)-\mathrm{Zr}(1)-\mathrm{O}(1)$ & 163.01(11) & N(7)-Zr(2)-Zr(3) & $45.12(7)$ \\
\hline $\mathrm{N}(2)-\mathrm{Zr}(1)-\mathrm{N}(4)$ & $96.81(12)$ & $\mathrm{N}(10)-\operatorname{Zr}(2)-\operatorname{Zr}(3)$ & 103.72(8) \\
\hline $\mathrm{N}(3)-\mathrm{Zr}(1)-\mathrm{N}(4)$ & $98.12(12)$ & $\mathrm{O}(2)-\operatorname{Zr}(2)-\operatorname{Zr}(3)$ & 46.89(5) \\
\hline $\mathrm{O}(1)-\mathrm{Zr}(1)-\mathrm{N}(4)$ & $77.84(9)$ & $\mathrm{N}(8)-\operatorname{Zr}(2)-\operatorname{Zr}(1)$ & 118.05(9) \\
\hline $\mathrm{N}(2)-\operatorname{Zr}(1)-\mathrm{N}(10)$ & $102.33(12)$ & $\mathrm{N}(9)-\operatorname{Zr}(2)-\operatorname{Zr}(1)$ & $125.93(11)$ \\
\hline $\mathrm{N}(3)-\mathrm{Zr}(1)-\mathrm{N}(10)$ & $102.66(12)$ & $\mathrm{O}(1)-\operatorname{Zr}(2)-\operatorname{Zr}(1)$ & $38.54(6)$ \\
\hline $\mathrm{O}(1)-\mathrm{Zr}(1)-\mathrm{N}(10)$ & 75.33(10) & $\mathrm{N}(7)-\operatorname{Zr}(2)-\operatorname{Zr}(1)$ & $104.70(7)$ \\
\hline $\mathrm{N}(4)-\mathrm{Zr}(1)-\mathrm{N}(10)$ & 148.41(10) & $\mathrm{N}(10)-\operatorname{Zr}(2)-\operatorname{Zr}(1)$ & $44.12(7)$ \\
\hline $\mathrm{N}(2)-\mathrm{Zr}(1)-\mathrm{O}(2)$ & 159.87(10) & $\mathrm{O}(2)-\operatorname{Zr}(2)-\operatorname{Zr}(1)$ & $46.62(5)$ \\
\hline $\mathrm{N}(3)-\mathrm{Zr}(1)-\mathrm{O}(2)$ & 98.65(11) & $\operatorname{Zr}(3)-\operatorname{Zr}(2)-\operatorname{Zr}(1)$ & $59.60(3)$ \\
\hline $\mathrm{O}(1)-\mathrm{Zr}(1)-\mathrm{O}(2)$ & $64.36(7)$ & $N(5)-Z r(3)-N(6)$ & $102.14(13)$ \\
\hline $\mathrm{N}(4)-\mathrm{Zr}(1)-\mathrm{O}(2)$ & 74.94(9) & $\mathrm{N}(5)-\mathrm{Zr}(3)-\mathrm{O}(1)$ & $96.06(11)$ \\
\hline $\mathrm{N}(10)-\mathrm{Zr}(1)-\mathrm{O}(2)$ & 78.60(9) & $\mathrm{N}(6)-\mathrm{Zr}(3)-\mathrm{O}(1)$ & $161.80(10)$ \\
\hline $\mathrm{N}(2)-\operatorname{Zr}(1)-\operatorname{Zr}(3)$ & 114.61(9) & $\mathrm{N}(5)-\mathrm{Zr}(3)-\mathrm{N}(4)$ & 95.67(11) \\
\hline $\mathrm{N}(3)-\operatorname{Zr}(1)-\operatorname{Zr}(3)$ & 128.80(10) & $\mathrm{N}(6)-\mathrm{Zr}(3)-\mathrm{N}(4)$ & 99.94(11) \\
\hline $\mathrm{O}(1)-\operatorname{Zr}(1)-\operatorname{Zr}(3)$ & $39.15(5)$ & $\mathrm{O}(1)-\mathrm{Zr}(3)-\mathrm{N}(4)$ & $78.10(9)$ \\
\hline N(4)-Zr(1)-Zr(3) & $44.25(7)$ & $\mathrm{N}(5)-\mathrm{Zr}(3)-\mathrm{N}(7)$ & $102.42(11)$ \\
\hline $\mathrm{N}(10)-\operatorname{Zr}(1)-\operatorname{Zr}(3)$ & $104.40(8)$ & $\mathrm{N}(6)-\mathrm{Zr}(3)-\mathrm{N}(7)$ & 99.88(11) \\
\hline $\mathrm{O}(2)-\operatorname{Zr}(1)-\operatorname{Zr}(3)$ & 47.05(6) & $\mathrm{O}(1)-\mathrm{Zr}(3)-\mathrm{N}(7)$ & $75.86(9)$ \\
\hline $\mathrm{N}(2)-\operatorname{Zr}(1)-\operatorname{Zr}(2)$ & 120.27(9) & $\mathrm{N}(4)-\mathrm{Zr}(3)-\mathrm{N}(7)$ & 149.59(9) \\
\hline $\mathrm{N}(3)-\operatorname{Zr}(1)-\operatorname{Zr}(2)$ & 129.83(10) & $\mathrm{N}(5)-\mathrm{Zr}(3)-\mathrm{O}(2)$ & $159.65(10)$ \\
\hline $\mathrm{O}(1)-\operatorname{Zr}(1)-\operatorname{Zr}(2)$ & $38.54(6)$ & $\mathrm{N}(6)-\mathrm{Zr}(3)-\mathrm{O}(2)$ & 97.39(10) \\
\hline $\mathrm{N}(4)-\operatorname{Zr}(1)-\operatorname{Zr}(2)$ & $103.90(7)$ & $\mathrm{O}(1)-\mathrm{Zr}(3)-\mathrm{O}(2)$ & $64.51(7)$ \\
\hline $\mathrm{N}(10)-\operatorname{Zr}(1)-\operatorname{Zr}(2)$ & $44.60(8)$ & $\mathrm{N}(4)-\mathrm{Zr}(3)-\mathrm{O}(2)$ & $75.21(8)$ \\
\hline $\mathrm{O}(2)-\operatorname{Zr}(1)-\operatorname{Zr}(2)$ & $47.25(5)$ & $\mathrm{N}(7)-\mathrm{Zr}(3)-\mathrm{O}(2)$ & 79.56(9) \\
\hline $\operatorname{Zr}(3)-\operatorname{Zr}(1)-\operatorname{Zr}(2)$ & 59.815(12) & $\mathrm{N}(5)-\operatorname{Zr}(3)-\operatorname{Zr}(1)$ & 113.93(9) \\
\hline $\mathrm{N}(8)-\operatorname{Zr}(2)-\mathrm{N}(9)$ & $100.27(15)$ & $\mathrm{N}(6)-\operatorname{Zr}(3)-\operatorname{Zr}(1)$ & 129.40(9) \\
\hline $\mathrm{N}(8)-\mathrm{Zr}(2)-\mathrm{O}(1)$ & 98.36(10) & $\mathrm{O}(1)-\operatorname{Zr}(3)-\operatorname{Zr}(1)$ & $39.23(6)$ \\
\hline $\mathrm{N}(9)-\mathrm{Zr}(2)-\mathrm{O}(1)$ & 160.89(13) & $\mathrm{N}(4)-\operatorname{Zr}(3)-\operatorname{Zr}(1)$ & $44.46(6)$ \\
\hline $\mathrm{N}(8)-\mathrm{Zr}(2)-\mathrm{N}(7)$ & 99.52(11) & $\mathrm{N}(7)-\operatorname{Zr}(3)-\operatorname{Zr}(1)$ & 105.32(7) \\
\hline $\mathrm{N}(9)-\mathrm{Zr}(2)-\mathrm{N}(7)$ & 104.77(14) & $\mathrm{O}(2)-\mathrm{Zr}(3)-\operatorname{Zr}(1)$ & $47.20(5)$ \\
\hline $\mathrm{O}(1)-\mathrm{Zr}(2)-\mathrm{N}(7)$ & 76.12(9) & $\mathrm{N}(5)-\operatorname{Zr}(3)-\operatorname{Zr}(2)$ & 120.59(9) \\
\hline $\mathrm{N}(8)-\operatorname{Zr}(2)-\mathrm{N}(10)$ & $96.07(12)$ & $\mathrm{N}(6)-\operatorname{Zr}(3)-\operatorname{Zr}(2)$ & 127.09(9) \\
\hline $\mathrm{N}(9)-\operatorname{Zr}(2)-\mathrm{N}(10)$ & $98.84(14)$ & $\mathrm{O}(1)-\operatorname{Zr}(3)-\operatorname{Zr}(2)$ & 39.07(6) \\
\hline $\mathrm{O}(1)-\mathrm{Zr}(2)-\mathrm{N}(10)$ & 74.91(9) & $\mathrm{N}(4)-\operatorname{Zr}(3)-\operatorname{Zr}(2)$ & $104.88(7)$ \\
\hline$N(7)-Z r(2)-N(10)$ & 148.74(10) & $\mathrm{N}(7)-\operatorname{Zr}(3)-\operatorname{Zr}(2)$ & 44.76(7) \\
\hline $\mathrm{N}(8)-\mathrm{Zr}(2)-\mathrm{O}(2)$ & 162.11(10) & $\mathrm{O}(2)-\operatorname{Zr}(3)-\operatorname{Zr}(2)$ & 47.67(5) \\
\hline $\mathrm{N}(9)-\mathrm{Zr}(2)-\mathrm{O}(2)$ & 97.28(13) & $\operatorname{Zr}(1)-\operatorname{Zr}(3)-\operatorname{Zr}(2)$ & $60.58(3)$ \\
\hline $\mathrm{O}(1)-\mathrm{Zr}(2)-\mathrm{O}(2)$ & $63.90(7)$ & & \\
\hline $\mathrm{N}(7)-\mathrm{Zr}(2)-\mathrm{O}(2)$ & $79.20(9)$ & & \\
\hline
\end{tabular}

Symmetry transformations used to generate equivalent atoms: 
Table S4. Anisotropic displacement parameters $\left(\AA^{2} \times 10^{3}\right)$ for 3 . The anisotropic displacement factor exponent takes the form: $-2 \pi^{2}\left[h^{2} a^{* 2} U^{11}+\ldots+2 h k a * b * U^{12}\right]$.

\begin{tabular}{|c|c|c|c|c|c|c|}
\hline & $\mathrm{U}^{11}$ & $\mathrm{U}^{22}$ & $\mathrm{U}^{33}$ & $\mathrm{U}^{23}$ & $\mathrm{U}^{13}$ & $\mathrm{U}^{12}$ \\
\hline $\mathrm{C}(1)$ & $67(3)$ & $50(2)$ & $28(2)$ & $-8(2)$ & $20(2)$ & $-12(2)$ \\
\hline C(2) & $41(2)$ & $45(2)$ & $35(2)$ & $-18(1)$ & $6(1)$ & $-1(1)$ \\
\hline C(3) & $55(2)$ & $53(2)$ & $37(2)$ & $-17(2)$ & $3(2)$ & $-9(2)$ \\
\hline C(4) & $51(2)$ & $62(3)$ & 61(3) & $-20(2)$ & $-2(2)$ & $-19(2)$ \\
\hline C(5) & $46(2)$ & $90(4)$ & 61(3) & $6(3)$ & $24(2)$ & $6(2)$ \\
\hline C(6) & $62(3)$ & $58(3)$ & $60(3)$ & $12(2)$ & $9(2)$ & $-15(2)$ \\
\hline $\mathrm{C}(7)$ & $40(2)$ & $50(2)$ & $35(2)$ & $5(2)$ & $-12(1)$ & $-6(2)$ \\
\hline C(8) & $28(2)$ & $46(2)$ & $53(2)$ & $-1(2)$ & $4(1)$ & $6(1)$ \\
\hline C(9) & $56(2)$ & $59(2)$ & $26(2)$ & $-3(1)$ & $6(2)$ & $4(2)$ \\
\hline$C(10)$ & $65(3)$ & $60(2)$ & $40(2)$ & $15(2)$ & $3(2)$ & $5(2)$ \\
\hline $\mathrm{C}(11)$ & $66(3)$ & $37(2)$ & 61(3) & $3(2)$ & $20(2)$ & $-6(2)$ \\
\hline$C(12)$ & $56(2)$ & $39(2)$ & $51(2)$ & $-6(2)$ & $11(2)$ & $12(2)$ \\
\hline$C(13)$ & $32(2)$ & $48(2)$ & $37(2)$ & $-2(1)$ & $12(1)$ & $-6(1)$ \\
\hline$C(14)$ & $43(2)$ & $50(2)$ & $54(2)$ & $-21(2)$ & $4(2)$ & $-16(2)$ \\
\hline$C(15)$ & $50(2)$ & $58(2)$ & $38(2)$ & $-14(2)$ & $3(2)$ & $19(2)$ \\
\hline$C(16)$ & $46(2)$ & $75(3)$ & $52(2)$ & $-4(2)$ & $-6(2)$ & $27(2)$ \\
\hline$C(17)$ & $52(3)$ & $112(5)$ & $45(2)$ & $-20(3)$ & $-6(2)$ & $-6(3)$ \\
\hline $\mathrm{C}(18)$ & $62(3)$ & 177(8) & $34(2)$ & $-7(3)$ & $-3(2)$ & $-9(4)$ \\
\hline $\mathrm{C}(19)$ & $51(2)$ & $34(2)$ & $53(2)$ & $-4(2)$ & $1(2)$ & $9(2)$ \\
\hline $\mathrm{C}(20)$ & $55(2)$ & $56(2)$ & $36(2)$ & $17(2)$ & $1(2)$ & $7(2)$ \\
\hline $\mathrm{N}(1)$ & $35(1)$ & $38(1)$ & $25(1)$ & $-11(1)$ & $9(1)$ & $0(1)$ \\
\hline $\mathrm{N}(2)$ & $39(2)$ & $37(1)$ & $34(1)$ & $-8(1)$ & $-3(1)$ & $-6(1)$ \\
\hline $\mathrm{N}(3)$ & $34(2)$ & $50(2)$ & $40(2)$ & $4(1)$ & $9(1)$ & $-6(1)$ \\
\hline $\mathrm{N}(4)$ & $26(1)$ & $33(1)$ & $29(1)$ & $1(1)$ & $-2(1)$ & $3(1)$ \\
\hline $\mathrm{N}(5)$ & $41(2)$ & $43(2)$ & $27(1)$ & $3(1)$ & $7(1)$ & $-1(1)$ \\
\hline $\mathrm{N}(6)$ & $46(2)$ & $28(1)$ & $40(2)$ & $-1(1)$ & $10(1)$ & $3(1)$ \\
\hline $\mathrm{N}(7)$ & $28(1)$ & $40(1)$ & $30(1)$ & $-10(1)$ & $7(1)$ & $-9(1)$ \\
\hline $\mathrm{N}(8)$ & $32(1)$ & $43(2)$ & $34(1)$ & $-4(1)$ & $4(1)$ & $10(1)$ \\
\hline $\mathrm{N}(9)$ & $53(2)$ & $83(3)$ & $26(1)$ & $-11(2)$ & $-3(1)$ & $-6(2)$ \\
\hline $\mathrm{N}(10)$ & $38(1)$ & $33(1)$ & $28(1)$ & $2(1)$ & $0(1)$ & $4(1)$ \\
\hline $\mathrm{O}(1)$ & $25(1)$ & $28(1)$ & 19(1) & $-4(1)$ & $1(1)$ & $2(1)$ \\
\hline $\mathrm{O}(2)$ & $27(1)$ & $31(1)$ & 21(1) & $-6(1)$ & $5(1)$ & $0(1)$ \\
\hline $\operatorname{Zr}(1)$ & $25(1)$ & $27(1)$ & $24(1)$ & $-1(1)$ & $2(1)$ & $-3(1)$ \\
\hline $\operatorname{Zr}(2)$ & $24(1)$ & $37(1)$ & $22(1)$ & $-6(1)$ & $0(1)$ & $2(1)$ \\
\hline $\operatorname{Zr}(3)$ & $27(1)$ & $26(1)$ & $25(1)$ & $-1(1)$ & $4(1)$ & $-1(1)$ \\
\hline
\end{tabular}


Table S5. Hydrogen coordinates $\left(\times 10^{4}\right)$ and isotropic displacement parameters $\left(\AA^{2} \times 10^{3}\right)$ for 3 .

\begin{tabular}{|c|c|c|c|c|}
\hline & $\mathrm{x}$ & $\mathrm{y}$ & $\mathrm{z}$ & $\mathrm{U}(\mathrm{eq})$ \\
\hline $\mathrm{H}(1 \mathrm{~A})$ & 2970 & 9464 & -1447 & 73 \\
\hline $\mathrm{H}(1 \mathrm{~B})$ & 2968 & 9889 & -913 & 73 \\
\hline $\mathrm{H}(1 \mathrm{C})$ & 2439 & 9727 & -1269 & 73 \\
\hline $\mathrm{H}(2 \mathrm{~A})$ & 2013 & 9073 & -956 & 60 \\
\hline $\mathrm{H}(2 \mathrm{~B})$ & 2236 & 8716 & -399 & 60 \\
\hline $\mathrm{H}(2 \mathrm{C})$ & 2499 & 8745 & -1136 & 60 \\
\hline $\mathrm{H}(3 \mathrm{~A})$ & 2727 & 10635 & 2421 & 73 \\
\hline $\mathrm{H}(3 \mathrm{~B})$ & 2243 & 10560 & 1921 & 73 \\
\hline $\mathrm{H}(3 \mathrm{C})$ & 2487 & 11072 & 2019 & 73 \\
\hline $\mathrm{H}(4 \mathrm{~A})$ & 3342 & 11238 & 1537 & 87 \\
\hline $\mathrm{H}(4 \mathrm{~B})$ & 3655 & 10817 & 1188 & 87 \\
\hline $\mathrm{H}(4 \mathrm{C})$ & 3547 & 10809 & 1990 & 87 \\
\hline $\mathrm{H}(5 \mathrm{~A})$ & 3798 & 10162 & -840 & 98 \\
\hline $\mathrm{H}(5 \mathrm{~B})$ & 3826 & 9758 & -276 & 98 \\
\hline $\mathrm{H}(5 \mathrm{C})$ & 4168 & 10223 & -191 & 98 \\
\hline $\mathrm{H}(6 \mathrm{~A})$ & 3778 & 10941 & -10 & 90 \\
\hline $\mathrm{H}(6 \mathrm{~B})$ & 3169 & 10983 & 138 & 90 \\
\hline $\mathrm{H}(6 \mathrm{C})$ & 3369 & 10869 & -616 & 90 \\
\hline $\mathrm{H}(7 \mathrm{~A})$ & 3343 & 9323 & 2105 & 62 \\
\hline $\mathrm{H}(7 \mathrm{~B})$ & 2987 & 9783 & 2128 & 62 \\
\hline $\mathrm{H}(7 \mathrm{C})$ & 3574 & 9818 & 1868 & 62 \\
\hline $\mathrm{H}(8 \mathrm{~A})$ & 3824 & 9461 & 836 & 63 \\
\hline $\mathrm{H}(8 \mathrm{~B})$ & 3404 & 9212 & 357 & 63 \\
\hline $\mathrm{H}(8 \mathrm{C})$ & 3568 & 8973 & 1063 & 63 \\
\hline $\mathrm{H}(9 \mathrm{~A})$ & 1713 & 9348 & 2878 & 70 \\
\hline $\mathrm{H}(9 \mathrm{~B})$ & 1854 & 9733 & 2314 & 70 \\
\hline $\mathrm{H}(9 \mathrm{C})$ & 2270 & 9596 & 2886 & 70 \\
\hline $\mathrm{H}(10 \mathrm{~A})$ & 2637 & 8856 & 2908 & 82 \\
\hline $\mathrm{H}(10 \mathrm{~B})$ & 2568 & 8501 & 2284 & 82 \\
\hline $\mathrm{H}(10 \mathrm{C})$ & 2102 & 8579 & 2811 & 82 \\
\hline $\mathrm{H}(11 \mathrm{~A})$ & 1985 & 7983 & 777 & 82 \\
\hline $\mathrm{H}(11 \mathrm{~B})$ & 1904 & 8316 & 1424 & 82 \\
\hline $\mathrm{H}(11 \mathrm{C})$ & 2360 & 7935 & 1422 & 82 \\
\hline $\mathrm{H}(12 \mathrm{~A})$ & 3108 & 8053 & 721 & 73 \\
\hline $\mathrm{H}(12 \mathrm{~B})$ & 3117 & 8484 & 197 & 73 \\
\hline $\mathrm{H}(12 \mathrm{C})$ & 2716 & 8062 & 89 & 73 \\
\hline $\mathrm{H}(13 \mathrm{~A})$ & 734 & 9253 & 1154 & 59 \\
\hline $\mathrm{H}(13 \mathrm{~B})$ & 1197 & 9454 & 1612 & 59 \\
\hline $\mathrm{H}(13 \mathrm{C})$ & 1103 & 8900 & 1561 & 59 \\
\hline $\mathrm{H}(14 \mathrm{~A})$ & 1321 & 8489 & 510 & 73 \\
\hline $\mathrm{H}(14 \mathrm{~B})$ & 1421 & 8802 & -154 & 73 \\
\hline $\mathrm{H}(14 \mathrm{C})$ & 872 & 8824 & 226 & 73 \\
\hline
\end{tabular}




\begin{tabular}{lrrrr}
$\mathrm{H}(15 \mathrm{~A})$ & 962 & 10731 & 1466 & 73 \\
$\mathrm{H}(15 \mathrm{~B})$ & 1278 & 10262 & 1635 & 73 \\
$\mathrm{H}(15 \mathrm{C})$ & 657 & 10255 & 1626 & 73 \\
$\mathrm{H}(16 \mathrm{~A})$ & 218 & 10399 & 542 & 87 \\
$\mathrm{H}(16 \mathrm{~B})$ & 560 & 10442 & -136 & 87 \\
$\mathrm{H}(16 \mathrm{C})$ & 580 & 10848 & 426 & 87 \\
$\mathrm{H}(17 \mathrm{~A})$ & 512 & 9798 & -959 & 105 \\
$\mathrm{H}(17 \mathrm{~B})$ & 663 & 9591 & -227 & 105 \\
$\mathrm{H}(17 \mathrm{C})$ & 792 & 9298 & -902 & 105 \\
$\mathrm{H}(18 \mathrm{~A})$ & 1359 & 9625 & -1707 & 137 \\
$\mathrm{H}(18 \mathrm{~B})$ & 1739 & 10047 & -1506 & 137 \\
$\mathrm{H}(18 \mathrm{C})$ & 1136 & 10148 & -1644 & 137 \\
$\mathrm{H}(19 \mathrm{~A})$ & 2161 & 11220 & 425 & 69 \\
$\mathrm{H}(19 B)$ & 1877 & 10871 & 943 & 69 \\
$\mathrm{H}(19 \mathrm{C})$ & 1576 & 11058 & 284 & 69 \\
$\mathrm{H}(20 \mathrm{~A})$ & 1846 & 10785 & -841 & 74 \\
$\mathrm{H}(20 \mathrm{~B})$ & 2364 & 10477 & -899 & 74 \\
$\mathrm{H}(20 \mathrm{C})$ & 2393 & 11006 & -628 & 74 \\
& & & & \\
\hline
\end{tabular}


Table S6. Crystal data and structure refinement for 4 .

\begin{tabular}{|c|c|c|}
\hline Empirical formula & \multicolumn{2}{|l|}{$\mathrm{C}_{20} \mathrm{H}_{60} \mathrm{~N}_{10} \mathrm{O}_{2} \mathrm{Hf}_{3}$} \\
\hline Formula weight & \multicolumn{2}{|l|}{1008.25} \\
\hline Temperature & \multicolumn{2}{|l|}{$-100(2){ }^{\circ} \mathrm{C}$} \\
\hline Wavelength & \multicolumn{2}{|l|}{$0.71073 \AA$} \\
\hline Crystal system & \multicolumn{2}{|l|}{ Orthorhombic } \\
\hline Space group & \multicolumn{2}{|l|}{ Fdd2 } \\
\hline \multirow[t]{3}{*}{ Unit cell dimensions } & $a=25.632(6) \AA$ & $\alpha=90^{\circ}$ \\
\hline & $\mathrm{b}=28.495(10) \AA$ & $\beta=90^{\circ}$ \\
\hline & $c=19.577(4) \AA$ & $\gamma=90^{\circ}$ \\
\hline Volume & $14299(7) \AA^{3}$ & \\
\hline $\mathrm{Z}$ & 16 & \\
\hline Density (calculated) & $1.873 \mathrm{~g} / \mathrm{cm}^{3}$ & \\
\hline Absorption coefficient & $8.720 \mathrm{~mm}^{-1}$ & \\
\hline $\mathrm{F}(000)$ & 7712 & \\
\hline Crystal size & $0.15 \times 0.10 \times 0.07$ & \\
\hline Theta range for data collection & 1.49 to $28.36^{\circ}$ & \\
\hline Index ranges & $-33 \leq \mathrm{h} \leq 34,-38 \leq$ &,$-25 \leq 1 \leq 25$ \\
\hline Reflections collected & 38108 & \\
\hline Independent reflections & $8638[\mathrm{R}($ int $)=0.0$ & \\
\hline Completeness to theta $=28.36^{\circ}$ & $98.8 \%$ & \\
\hline Absorption correction & Semi-empirical frc & valents \\
\hline Max. and min. transmission & 0.5804 and 0.3546 & \\
\hline Refinement method & Full-matrix least-s & on $\mathrm{F}^{2}$ \\
\hline Data / restraints / parameters & 8638 / 1 / 336 & \\
\hline Goodness-of-fit on $\mathrm{F}^{2}$ & 1.080 & \\
\hline Final R indices [I > 2sigma(I)] & $\mathrm{R} 1=0.0299, \mathrm{wR} 2$ & \\
\hline $\mathrm{R}$ indices (all data) & $\mathrm{R} 1=0.0318, \mathrm{wR} 2$ & \\
\hline Absolute structure parameter & $1.008(16)$ & \\
\hline Largest diff. peak and hole & 1.416 and -0.597 & \\
\hline
\end{tabular}


Table S7. Atomic coordinates $\left(\times 10^{4}\right)$ and equivalent isotropic displacement parameters $\left(\AA^{2} \times\right.$ $10^{3}$ ) for 4 . $U(e q)$ is defined as one third of the trace of the orthogonalized Uij tensor.

\begin{tabular}{|c|c|c|c|c|}
\hline & $\mathrm{X}$ & $\mathrm{y}$ & $\mathrm{z}$ & $\mathrm{U}(\mathrm{eq})$ \\
\hline $\mathrm{C}(1)$ & $2209(4)$ & $9618(4)$ & 2132(5) & $43(2)$ \\
\hline $\mathrm{C}(2)$ & 2670(3) & 8937(3) & 1831(4) & $33(2)$ \\
\hline C(3) & 1164(4) & 10119(4) & 1377(6) & $55(3)$ \\
\hline C(4) & $1570(5)$ & $10837(4)$ & 1187(6) & 51(3) \\
\hline C(5) & $1605(4)$ & 10916(5) & $-489(7)$ & 57(3) \\
\hline C(6) & $2446(4)$ & $10741(4)$ & $-956(5)$ & $44(2)$ \\
\hline C(7) & $2815(4)$ & 10709(4) & 1685(5) & $43(2)$ \\
\hline C(8) & $3094(4)$ & 10956(3) & 580(5) & $39(2)$ \\
\hline C(9) & $3620(5)$ & $9914(6)$ & $2500(6)$ & $70(4)$ \\
\hline $\mathrm{C}(10)$ & $4250(4)$ & 9623(5) & $1754(6)$ & $55(3)$ \\
\hline $\mathrm{C}(11)$ & $4035(4)$ & 10382(4) & $-379(5)$ & $44(2)$ \\
\hline $\mathrm{C}(12)$ & $4446(4)$ & $10509(4)$ & 693(5) & $50(3)$ \\
\hline C(13) & $3746(4)$ & 8801(3) & 777(6) & $42(2)$ \\
\hline$C(14)$ & $3907(4)$ & $9205(3)$ & $-265(5)$ & $36(2)$ \\
\hline C(15) & $2109(4)$ & 8264(3) & 617(6) & $44(2)$ \\
\hline $\mathrm{C}(16)$ & $2844(4)$ & 8150(3) & $-90(6)$ & $48(2)$ \\
\hline $\mathrm{C}(17)$ & $3015(4)$ & $9465(4)$ & $-1542(4)$ & $42(2)$ \\
\hline C(18) & $2597(5)$ & $8724(4)$ & $-1507(5)$ & $49(2)$ \\
\hline C(19) & 1493(3) & $9276(3)$ & 222(5) & $36(2)$ \\
\hline C(20) & $1738(4)$ & 9622(3) & $-833(5)$ & $37(2)$ \\
\hline $\mathrm{Hf}(1)$ & 2224(1) & 10189(1) & 398(1) & 21(1) \\
\hline $\operatorname{Hf}(2)$ & $3428(1)$ & 9911(1) & 803(1) & $24(1)$ \\
\hline Hf(3) & 2704(1) & 9183(1) & $-64(1)$ & $22(1)$ \\
\hline $\mathrm{N}(1)$ & 2316(3) & 9292(2) & $1577(4)$ & $29(1)$ \\
\hline $\mathrm{N}(2)$ & 1593(3) & 10349(3) & $1025(4)$ & $38(2)$ \\
\hline $\mathrm{N}(3)$ & 2084(3) & $10652(2)$ & $-378(4)$ & $30(1)$ \\
\hline $\mathrm{N}(4)$ & 2890(3) & $10548(2)$ & 975(3) & $28(1)$ \\
\hline $\mathrm{N}(5)$ & 3732(3) & 9840(3) & $1780(4)$ & $39(2)$ \\
\hline $\mathrm{N}(6)$ & 4021(3) & $10268(2)$ & $344(4)$ & $31(1)$ \\
\hline $\mathrm{N}(7)$ & $3548(3)$ & $9188(2)$ & $333(4)$ & $30(1)$ \\
\hline $\mathrm{N}(8)$ & 2503(3) & $8509(2)$ & $200(4)$ & $33(2)$ \\
\hline N(9) & 2802(3) & 9112(3) & $-1090(4)$ & $33(2)$ \\
\hline $\mathrm{N}(10)$ & 1914(3) & $9533(2)$ & $-127(3)$ & $26(1)$ \\
\hline $\mathrm{O}(1)$ & 2591(2) & $9546(2)$ & 1025(3) & $23(1)$ \\
\hline $\mathrm{O}(2)$ & $2905(2)$ & $9898(2)$ & $-31(3)$ & $20(1)$ \\
\hline
\end{tabular}


Table S8. Bond lengths $(\AA)$ and angles $\left(^{\circ}\right)$ for 4.

\begin{tabular}{|c|c|c|c|}
\hline $\mathrm{C}(1)-\mathrm{N}(1)$ & $1.458(12)$ & Hf(1)-N(3) & $2.044(7)$ \\
\hline $\mathrm{C}(2)-\mathrm{N}(1)$ & $1.446(11)$ & Hf(1)-N(2) & $2.081(7)$ \\
\hline $\mathrm{C}(3)-\mathrm{N}(2)$ & $1.454(13)$ & $\mathrm{Hf}(1)-\mathrm{O}(2)$ & $2.108(5)$ \\
\hline $\mathrm{C}(4)-\mathrm{N}(2)$ & $1.428(14)$ & Hf(1)-N(10) & $2.275(6)$ \\
\hline $\mathrm{C}(5)-\mathrm{N}(3)$ & $1.457(12)$ & $\mathrm{Hf}(1)-\mathrm{N}(4)$ & $2.289(7)$ \\
\hline $\mathrm{C}(6)-\mathrm{N}(3)$ & $1.485(12)$ & $\mathrm{Hf}(1)-\mathrm{O}(1)$ & $2.399(5)$ \\
\hline$C(7)-N(4)$ & $1.476(11)$ & $\mathrm{Hf}(2)-\mathrm{N}(6)$ & $2.037(6)$ \\
\hline $\mathrm{C}(8)-\mathrm{N}(4)$ & $1.490(10)$ & Hf(2)-N(5) & $2.075(7)$ \\
\hline $\mathrm{C}(9)-\mathrm{N}(5)$ & $1.453(14)$ & $\mathrm{Hf}(2)-\mathrm{O}(2)$ & $2.112(5)$ \\
\hline C(10)-N(5) & $1.466(13)$ & Hf(2)-N(7) & $2.279(7)$ \\
\hline $\mathrm{C}(11)-\mathrm{N}(6)$ & $1.453(12)$ & $\mathrm{Hf}(2)-\mathrm{N}(4)$ & $2.302(7)$ \\
\hline $\mathrm{C}(12)-\mathrm{N}(6)$ & $1.456(11)$ & $\mathrm{Hf}(2)-\mathrm{O}(1)$ & $2.423(5)$ \\
\hline $\mathrm{C}(13)-\mathrm{N}(7)$ & $1.494(10)$ & Hf(3)-N(9) & $2.036(7)$ \\
\hline $\mathrm{C}(14)-\mathrm{N}(7)$ & $1.490(10)$ & Hf(3)-N(8) & $2.054(7)$ \\
\hline C(15)-N(8) & $1.474(11)$ & $\mathrm{Hf}(3)-\mathrm{O}(2)$ & $2.102(5)$ \\
\hline C(16)-N(8) & $1.461(11)$ & Hf(3)-N(10) & $2.260(6)$ \\
\hline $\mathrm{C}(17)-\mathrm{N}(9)$ & $1.444(12)$ & $\mathrm{Hf}(3)-\mathrm{N}(7)$ & $2.299(7)$ \\
\hline $\mathrm{C}(18)-\mathrm{N}(9)$ & $1.470(12)$ & $\mathrm{Hf}(3)-\mathrm{O}(1)$ & $2.386(5)$ \\
\hline $\mathrm{C}(19)-\mathrm{N}(10)$ & $1.471(11)$ & $\mathrm{N}(1)-\mathrm{O}(1)$ & $1.479(8)$ \\
\hline $\mathrm{C}(20)-\mathrm{N}(10)$ & $1.478(11)$ & & \\
\hline $\mathrm{N}(3)-\mathrm{Hf}(1)-\mathrm{N}(2)$ & $99.2(3)$ & $\mathrm{N}(5)-\mathrm{Hf}(2)-\mathrm{N}(4)$ & 99.6(3) \\
\hline $\mathrm{N}(3)-\mathrm{Hf}(1)-\mathrm{O}(2)$ & $95.9(2)$ & $\mathrm{O}(2)-\mathrm{Hf}(2)-\mathrm{N}(4)$ & $75.4(2)$ \\
\hline $\mathrm{N}(2)-\mathrm{Hf}(1)-\mathrm{O}(2)$ & 164.8(3) & N(7)-Hf(2)-N(4) & $148.5(2)$ \\
\hline $\mathrm{N}(3)-\mathrm{Hf}(1)-\mathrm{N}(10)$ & 97.7(3) & $\mathrm{N}(6)-\mathrm{Hf}(2)-\mathrm{O}(1)$ & $162.5(2)$ \\
\hline $\mathrm{N}(2)-\mathrm{Hf}(1)-\mathrm{N}(10)$ & 100.1(3) & $\mathrm{N}(5)-\mathrm{Hf}(2)-\mathrm{O}(1)$ & $97.2(3)$ \\
\hline $\mathrm{O}(2)-\mathrm{Hf}(1)-\mathrm{N}(10)$ & $77.6(2)$ & $\mathrm{O}(2)-\mathrm{Hf}(2)-\mathrm{O}(1)$ & $64.49(18)$ \\
\hline $\mathrm{N}(3)-\mathrm{Hf}(1)-\mathrm{N}(4)$ & 102.1(3) & $\mathrm{N}(7)-\mathrm{Hf}(2)-\mathrm{O}(1)$ & $78.6(2)$ \\
\hline $\mathrm{N}(2)-\mathrm{Hf}(1)-\mathrm{N}(4)$ & 101.0(3) & $\mathrm{N}(4)-\mathrm{Hf}(2)-\mathrm{O}(1)$ & $77.5(2)$ \\
\hline $\mathrm{O}(2)-\mathrm{Hf}(1)-\mathrm{N}(4)$ & 75.8(2) & N(9)-Hf(3)-N(8) & 100.7(3) \\
\hline $\mathrm{N}(10)-\mathrm{Hf}(1)-\mathrm{N}(4)$ & $148.2(2)$ & $\mathrm{N}(9)-\mathrm{Hf}(3)-\mathrm{O}(2)$ & 95.5(3) \\
\hline $\mathrm{N}(3)-\mathrm{Hf}(1)-\mathrm{O}(1)$ & $160.5(2)$ & N(8)-Hf(3)-O(2) & 163.7(3) \\
\hline $\mathrm{N}(2)-\mathrm{Hf}(1)-\mathrm{O}(1)$ & 99.9(3) & $\mathrm{N}(9)-\mathrm{Hf}(3)-\mathrm{N}(10)$ & 95.8(3) \\
\hline $\mathrm{O}(2)-\mathrm{Hf}(1)-\mathrm{O}(1)$ & $65.01(17)$ & $\mathrm{N}(8)-\mathrm{Hf}(3)-\mathrm{N}(10)$ & 101.6(3) \\
\hline $\mathrm{N}(10)-\mathrm{Hf}(1)-\mathrm{O}(1)$ & $74.9(2)$ & $\mathrm{O}(2)-\mathrm{Hf}(3)-\mathrm{N}(10)$ & 78.1(2) \\
\hline $\mathrm{N}(4)-\mathrm{Hf}(1)-\mathrm{O}(1)$ & 78.2(2) & $\mathrm{N}(9)-\mathrm{Hf}(3)-\mathrm{N}(7)$ & 102.6(3) \\
\hline $\mathrm{N}(6)-\mathrm{Hf}(2)-\mathrm{N}(5)$ & 100.0(3) & $\mathrm{N}(8)-\mathrm{Hf}(3)-\mathrm{N}(7)$ & 99.1(3) \\
\hline $\mathrm{N}(6)-\mathrm{Hf}(2)-\mathrm{O}(2)$ & $98.2(2)$ & $\mathrm{O}(2)-\mathrm{Hf}(3)-\mathrm{N}(7)$ & $75.7(2)$ \\
\hline $\mathrm{N}(5)-\mathrm{Hf}(2)-\mathrm{O}(2)$ & 161.6(3) & $\mathrm{N}(10)-\mathrm{Hf}(3)-\mathrm{N}(7)$ & $149.1(2)$ \\
\hline $\mathrm{N}(6)-\mathrm{Hf}(2)-\mathrm{N}(7)$ & 99.9(3) & $\mathrm{N}(9)-\mathrm{Hf}(3)-\mathrm{O}(1)$ & $160.0(2)$ \\
\hline $\mathrm{N}(5)-\mathrm{Hf}(2)-\mathrm{N}(7)$ & 103.5(3) & $\mathrm{N}(8)-\mathrm{Hf}(3)-\mathrm{O}(1)$ & $98.6(2)$ \\
\hline $\mathrm{O}(2)-\mathrm{Hf}(2)-\mathrm{N}(7)$ & 75.9(2) & $\mathrm{O}(2)-\mathrm{Hf}(3)-\mathrm{O}(1)$ & $65.33(18)$ \\
\hline $\mathrm{N}(6)-\mathrm{Hf}(2)-\mathrm{N}(4)$ & $96.8(3)$ & $\mathrm{N}(10)-\mathrm{Hf}(3)-\mathrm{O}(1)$ & $75.5(2)$ \\
\hline $\mathrm{N}(7)-\mathrm{Hf}(3)-\mathrm{O}(1)$ & $79.0(2)$ & $\mathrm{C}(2)-\mathrm{N}(1)-\mathrm{C}(1)$ & $108.0(7)$ \\
\hline
\end{tabular}




\begin{tabular}{|c|c|c|c|}
\hline $\mathrm{C}(2)-\mathrm{N}(1)-\mathrm{O}(1)$ & 107.1(6) & C(13)-N(7)-Hf(3) & $120.8(5)$ \\
\hline $\mathrm{C}(1)-\mathrm{N}(1)-\mathrm{O}(1)$ & $108.8(6)$ & $\mathrm{Hf}(2)-\mathrm{N}(7)-\mathrm{Hf}(3)$ & $90.9(2)$ \\
\hline C(4)-N(2)-C(3) & 107.6(8) & $\mathrm{C}(16)-\mathrm{N}(8)-\mathrm{C}(15)$ & 107.1(7) \\
\hline $\mathrm{C}(4)-\mathrm{N}(2)-\mathrm{Hf}(1)$ & $112.1(7)$ & $\mathrm{C}(16)-\mathrm{N}(8)-\mathrm{Hf}(3)$ & $114.0(6)$ \\
\hline $\mathrm{C}(3)-\mathrm{N}(2)-\mathrm{Hf}(1)$ & $140.2(7)$ & C(15)-N(8)-Hf(3) & $139.0(6)$ \\
\hline $\mathrm{C}(5)-\mathrm{N}(3)-\mathrm{C}(6)$ & 109.0(8) & $\mathrm{C}(17)-\mathrm{N}(9)-\mathrm{C}(18)$ & 108.6(8) \\
\hline $\mathrm{C}(5)-\mathrm{N}(3)-\mathrm{Hf}(1)$ & $126.3(7)$ & $\mathrm{C}(17)-\mathrm{N}(9)-\mathrm{Hf}(3)$ & $125.6(6)$ \\
\hline $\mathrm{C}(6)-\mathrm{N}(3)-\mathrm{Hf}(1)$ & $124.6(6)$ & C(18)-N(9)-Hf(3) & $125.3(6)$ \\
\hline $\mathrm{C}(7)-\mathrm{N}(4)-\mathrm{C}(8)$ & $107.0(7)$ & $\mathrm{C}(19)-\mathrm{N}(10)-\mathrm{C}(20)$ & $107.2(7)$ \\
\hline $\mathrm{C}(7)-\mathrm{N}(4)-\mathrm{Hf}(1)$ & $120.4(6)$ & C(19)-N(10)-Hf(3) & $114.3(5)$ \\
\hline $\mathrm{C}(8)-\mathrm{N}(4)-\mathrm{Hf}(1)$ & $110.7(5)$ & $\mathrm{C}(20)-\mathrm{N}(10)-\mathrm{Hf}(3)$ & $113.6(5)$ \\
\hline $\mathrm{C}(7)-\mathrm{N}(4)-\mathrm{Hf}(2)$ & $117.4(6)$ & $\mathrm{C}(19)-\mathrm{N}(10)-\mathrm{Hf}(1)$ & $117.1(5)$ \\
\hline C(8)-N(4)-Hf(2) & $109.2(5)$ & $\mathrm{C}(20)-\mathrm{N}(10)-\mathrm{Hf}(1)$ & $112.8(5)$ \\
\hline $\mathrm{Hf}(1)-\mathrm{N}(4)-\mathrm{Hf}(2)$ & $91.3(2)$ & Hf(3)-N(10)-Hf(1) & $91.5(2)$ \\
\hline $\mathrm{C}(9)-\mathrm{N}(5)-\mathrm{C}(10)$ & 105.8(8) & $\mathrm{N}(1)-\mathrm{O}(1)-\mathrm{Hf}(3)$ & $119.9(4)$ \\
\hline C(9)-N(5)-Hf(2) & $143.7(7)$ & N(1)-O(1)-Hf(1) & $124.1(4)$ \\
\hline $\mathrm{C}(10)-\mathrm{N}(5)-\mathrm{Hf}(2)$ & $110.5(6)$ & $\mathrm{Hf}(3)-\mathrm{O}(1)-\mathrm{Hf}(1)$ & $85.50(16)$ \\
\hline $\mathrm{C}(11)-\mathrm{N}(6)-\mathrm{C}(12)$ & $109.4(7)$ & $\mathrm{N}(1)-\mathrm{O}(1)-\mathrm{Hf}(2)$ & $139.7(4)$ \\
\hline $\mathrm{C}(11)-\mathrm{N}(6)-\mathrm{Hf}(2)$ & $124.0(6)$ & $\mathrm{Hf}(3)-\mathrm{O}(1)-\mathrm{Hf}(2)$ & $85.36(16)$ \\
\hline $\mathrm{C}(12)-\mathrm{N}(6)-\mathrm{Hf}(2)$ & $125.9(6)$ & $\mathrm{Hf}(1)-\mathrm{O}(1)-\mathrm{Hf}(2)$ & 85.81(16) \\
\hline $\mathrm{C}(14)-\mathrm{N}(7)-\mathrm{C}(13)$ & $105.7(6)$ & $\mathrm{Hf}(3)-\mathrm{O}(2)-\mathrm{Hf}(1)$ & 100.99(19) \\
\hline $\mathrm{C}(14)-\mathrm{N}(7)-\mathrm{Hf}(2)$ & $111.7(5)$ & $\mathrm{Hf}(3)-\mathrm{O}(2)-\mathrm{Hf}(2)$ & $101.4(2)$ \\
\hline $\mathrm{C}(13)-\mathrm{N}(7)-\mathrm{Hf}(2)$ & $118.6(6)$ & $\mathrm{Hf}(1)-\mathrm{O}(2)-\mathrm{Hf}(2)$ & 102.1(2) \\
\hline $\mathrm{C}(14)-\mathrm{N}(7)-\mathrm{Hf}(3)$ & $108.5(5)$ & & \\
\hline
\end{tabular}

Symmetry transformations used to generate equivalent atoms: 
Table S9. Anisotropic displacement parameters $\left(\AA^{2} \times 10^{3}\right)$ for 4 . The anisotropic displacement factor exponent takes the form: $-2 \pi^{2}\left[h^{2} a^{* 2} U^{11}+\ldots+2 h k a * b * U^{12}\right]$.

\begin{tabular}{|c|c|c|c|c|c|c|}
\hline & $\mathrm{U}^{11}$ & $\mathrm{U}^{22}$ & $\mathrm{U}^{33}$ & $\mathrm{U}^{23}$ & $\mathrm{U}^{13}$ & $\mathrm{U}^{12}$ \\
\hline $\mathrm{C}(1)$ & $54(6)$ & $49(5)$ & $26(4)$ & $6(4)$ & $16(4)$ & $10(4)$ \\
\hline $\mathrm{C}(2)$ & $34(4)$ & $38(4)$ & $26(4)$ & 15(3) & $2(3)$ & $0(3)$ \\
\hline C(3) & $41(6)$ & $73(8)$ & $52(7)$ & $-4(6)$ & $20(5)$ & $0(5)$ \\
\hline C(4) & $47(6)$ & $53(6)$ & $54(7)$ & $-9(5)$ & $8(5)$ & $15(5)$ \\
\hline C(5) & $44(6)$ & $63(7)$ & $64(7)$ & $23(6)$ & $-4(5)$ & $18(5)$ \\
\hline C(6) & $56(6)$ & $43(5)$ & $32(5)$ & $16(4)$ & $2(4)$ & $5(4)$ \\
\hline $\mathrm{C}(7)$ & $47(5)$ & $51(5)$ & $31(5)$ & $-15(4)$ & $-1(4)$ & $-9(4)$ \\
\hline C(8) & $50(6)$ & $25(4)$ & $44(5)$ & $3(3)$ & $-1(4)$ & $-6(4)$ \\
\hline$C(9)$ & $46(7)$ & $134(13)$ & $30(5)$ & $3(7)$ & $-4(5)$ & $2(7)$ \\
\hline $\mathrm{C}(10)$ & $38(5)$ & 85(8) & $42(6)$ & $19(5)$ & $-9(4)$ & $7(5)$ \\
\hline $\mathrm{C}(11)$ & $44(5)$ & $48(5)$ & $40(5)$ & $12(4)$ & $3(4)$ & $-16(4)$ \\
\hline$C(12)$ & $38(5)$ & $65(6)$ & $45(6)$ & $3(5)$ & $-8(4)$ & $-27(5)$ \\
\hline C(13) & $34(4)$ & $37(4)$ & $54(6)$ & $18(4)$ & $5(4)$ & $9(3)$ \\
\hline $\mathrm{C}(14)$ & $29(4)$ & $43(5)$ & $36(5)$ & $2(4)$ & $10(3)$ & $7(3)$ \\
\hline C(15) & $47(6)$ & $35(4)$ & $50(6)$ & $3(4)$ & $10(4)$ & $-11(4)$ \\
\hline$C(16)$ & $60(6)$ & $26(4)$ & $59(6)$ & $-3(4)$ & $17(6)$ & $4(4)$ \\
\hline C(17) & $49(6)$ & $55(6)$ & $23(4)$ & $3(4)$ & $3(4)$ & $-3(4)$ \\
\hline C(18) & $61(6)$ & $46(5)$ & $39(5)$ & $-16(4)$ & $7(4)$ & $-8(5)$ \\
\hline C(19) & $23(4)$ & $38(4)$ & $48(5)$ & $-4(4)$ & $2(3)$ & $-4(3)$ \\
\hline$C(20)$ & $33(4)$ & $44(5)$ & $33(4)$ & $-5(4)$ & $-10(3)$ & $3(4)$ \\
\hline Hf(1) & $20(1)$ & $22(1)$ & $22(1)$ & $1(1)$ & $1(1)$ & $3(1)$ \\
\hline $\mathrm{Hf}(2)$ & $21(1)$ & $29(1)$ & 21(1) & $5(1)$ & $0(1)$ & $-1(1)$ \\
\hline Hf(3) & $22(1)$ & $20(1)$ & $23(1)$ & $1(1)$ & $3(1)$ & $1(1)$ \\
\hline $\mathrm{N}(1)$ & $34(4)$ & $29(3)$ & $23(3)$ & $8(3)$ & $9(3)$ & $-2(3)$ \\
\hline $\mathrm{N}(2)$ & $29(4)$ & $51(4)$ & $34(4)$ & $-4(3)$ & $10(3)$ & $8(3)$ \\
\hline $\mathrm{N}(3)$ & 31(3) & 28(3) & $31(4)$ & $5(3)$ & $0(3)$ & $4(3)$ \\
\hline $\mathrm{N}(4)$ & $32(3)$ & $23(3)$ & 28(3) & $-3(3)$ & $0(3)$ & $-6(2)$ \\
\hline $\mathrm{N}(5)$ & $29(4)$ & $64(5)$ & $24(4)$ & $7(3)$ & $-5(3)$ & $-1(3)$ \\
\hline $\mathrm{N}(6)$ & $27(3)$ & $37(3)$ & $30(3)$ & $4(3)$ & $2(3)$ & $-9(3)$ \\
\hline $\mathrm{N}(7)$ & $24(3)$ & $34(3)$ & 31(3) & $10(3)$ & 8(3) & $8(2)$ \\
\hline $\mathrm{N}(8)$ & $38(4)$ & $23(3)$ & $38(4)$ & $-2(3)$ & $6(3)$ & $-2(3)$ \\
\hline N(9) & $34(4)$ & $40(4)$ & 25(3) & $-1(3)$ & $3(3)$ & $-3(3)$ \\
\hline $\mathrm{N}(10)$ & $26(3)$ & $26(3)$ & $24(3)$ & $-1(3)$ & $-3(3)$ & $0(2)$ \\
\hline $\mathrm{O}(1)$ & $21(2)$ & $26(2)$ & $21(2)$ & $8(2)$ & $6(2)$ & $1(2)$ \\
\hline $\mathrm{O}(2)$ & $17(2)$ & $22(2)$ & $21(2)$ & $5(2)$ & $4(2)$ & $-3(2)$ \\
\hline
\end{tabular}


Table S10. Hydrogen coordinates $\left(\times 10^{4}\right)$ and isotropic displacement parameters $\left(\AA^{2} \times 10^{3}\right)$ for 4.

\begin{tabular}{|c|c|c|c|c|}
\hline & $\mathrm{x}$ & $\mathrm{y}$ & $\mathrm{Z}$ & $\mathrm{U}(\mathrm{eq})$ \\
\hline $\mathrm{H}(1 \mathrm{~A})$ & 2003 & 9460 & 2485 & 65 \\
\hline $\mathrm{H}(1 \mathrm{~B})$ & 2012 & 9887 & 1955 & 65 \\
\hline $\mathrm{H}(1 \mathrm{C})$ & 2539 & 9728 & 2329 & 65 \\
\hline $\mathrm{H}(2 \mathrm{~A})$ & 2997 & 9085 & 1972 & 49 \\
\hline $\mathrm{H}(2 \mathrm{~B})$ & 2741 & 8708 & 1469 & 49 \\
\hline $\mathrm{H}(2 \mathrm{C})$ & 2511 & 8777 & 2222 & 49 \\
\hline $\mathrm{H}(3 \mathrm{~A})$ & 1195 & 10174 & 1870 & 83 \\
\hline $\mathrm{H}(3 \mathrm{~B})$ & 1175 & 9781 & 1286 & 83 \\
\hline $\mathrm{H}(3 \mathrm{C})$ & 832 & 10248 & 1214 & 83 \\
\hline $\mathrm{H}(4 \mathrm{~A})$ & 1272 & 10981 & 953 & 77 \\
\hline $\mathrm{H}(4 \mathrm{~B})$ & 1893 & 10990 & 1038 & 77 \\
\hline $\mathrm{H}(4 \mathrm{C})$ & 1530 & 10876 & 1681 & 77 \\
\hline $\mathrm{H}(5 \mathrm{~A})$ & 1660 & 11244 & -359 & 85 \\
\hline $\mathrm{H}(5 \mathrm{~B})$ & 1325 & 10782 & -209 & 85 \\
\hline $\mathrm{H}(5 \mathrm{C})$ & 1507 & 10899 & -972 & 85 \\
\hline $\mathrm{H}(6 \mathrm{~A})$ & 2271 & 10665 & -1388 & 66 \\
\hline $\mathrm{H}(6 \mathrm{~B})$ & 2757 & 10544 & -905 & 66 \\
\hline $\mathrm{H}(6 \mathrm{C})$ & 2548 & 11072 & -957 & 66 \\
\hline $\mathrm{H}(7 \mathrm{~A})$ & 3156 & 10771 & 1893 & 65 \\
\hline $\mathrm{H}(7 \mathrm{~B})$ & 2634 & 10465 & 1946 & 65 \\
\hline $\mathrm{H}(7 \mathrm{C})$ & 2606 & 10997 & 1686 & 65 \\
\hline $\mathrm{H}(8 \mathrm{~A})$ & 2845 & 11216 & 608 & 59 \\
\hline $\mathrm{H}(8 \mathrm{~B})$ & 3140 & 10865 & 101 & 59 \\
\hline $\mathrm{H}(8 \mathrm{C})$ & 3430 & 11054 & 770 & 59 \\
\hline $\mathrm{H}(9 \mathrm{~A})$ & 3604 & 9610 & 2733 & 105 \\
\hline $\mathrm{H}(9 \mathrm{~B})$ & 3284 & 10075 & 2548 & 105 \\
\hline $\mathrm{H}(9 \mathrm{C})$ & 3896 & 10105 & 2704 & 105 \\
\hline $\mathrm{H}(10 \mathrm{~A})$ & 4501 & 9824 & 1992 & 83 \\
\hline $\mathrm{H}(10 \mathrm{~B})$ & 4357 & 9585 & 1277 & 83 \\
\hline $\mathrm{H}(10 \mathrm{C})$ & 4238 & 9315 & 1977 & 83 \\
\hline $\mathrm{H}(11 \mathrm{~A})$ & 3932 & 10710 & -443 & 66 \\
\hline $\mathrm{H}(11 \mathrm{~B})$ & 3793 & 10177 & -626 & 66 \\
\hline $\mathrm{H}(11 \mathrm{C})$ & 4390 & 10336 & -554 & 66 \\
\hline $\mathrm{H}(12 \mathrm{~A})$ & 4780 & 10391 & 522 & 74 \\
\hline $\mathrm{H}(12 \mathrm{~B})$ & 4421 & 10450 & 1185 & 74 \\
\hline $\mathrm{H}(12 \mathrm{C})$ & 4421 & 10847 & 606 & 74 \\
\hline$H(13 A)$ & 3673 & 8498 & 560 & 62 \\
\hline $\mathrm{H}(13 \mathrm{~B})$ & 3572 & 8814 & 1222 & 62 \\
\hline $\mathrm{H}(13 \mathrm{C})$ & 4123 & 8836 & 840 & 62 \\
\hline $\mathrm{H}(14 \mathrm{~A})$ & 4253 & 9310 & -114 & 54 \\
\hline $\mathrm{H}(14 \mathrm{~B})$ & 3771 & 9426 & -605 & 54 \\
\hline
\end{tabular}




\begin{tabular}{lrrrr}
$\mathrm{H}(14 \mathrm{C})$ & 3935 & 8892 & -468 & 54 \\
$\mathrm{H}(15 \mathrm{~A})$ & 1860 & 8104 & 316 & 66 \\
$\mathrm{H}(15 \mathrm{~B})$ & 1923 & 8492 & 901 & 66 \\
$\mathrm{H}(15 \mathrm{C})$ & 2282 & 8032 & 910 & 66 \\
$\mathrm{H}(16 \mathrm{~A})$ & 3006 & 7970 & 280 & 72 \\
$\mathrm{H}(16 \mathrm{~B})$ & 3117 & 8301 & -364 & 72 \\
$\mathrm{H}(16 \mathrm{C})$ & 2640 & 7939 & -380 & 72 \\
$\mathrm{H}(17 \mathrm{~A})$ & 3300 & 9329 & -1809 & 64 \\
$\mathrm{H}(17 \mathrm{~B})$ & 3147 & 9728 & -1271 & 64 \\
$\mathrm{H}(17 \mathrm{C})$ & 2741 & 9576 & -1851 & 64 \\
$\mathrm{H}(18 \mathrm{~A})$ & 2399 & 8852 & -1892 & 73 \\
$\mathrm{H}(18 B)$ & 2368 & 8528 & -1226 & 73 \\
$\mathrm{H}(18 \mathrm{C})$ & 2887 & 8535 & -1679 & 73 \\
$\mathrm{H}(19 \mathrm{~A})$ & 1173 & 9464 & 212 & 55 \\
$\mathrm{H}(19 B)$ & 1594 & 9217 & 697 & 55 \\
$\mathrm{H}(19 \mathrm{C})$ & 1433 & 8977 & -11 & 55 \\
$\mathrm{H}(20 \mathrm{~A})$ & 1633 & 9325 & -1046 & 55 \\
$\mathrm{H}(20 B)$ & 2023 & 9763 & -1096 & 55 \\
$\mathrm{H}(20 \mathrm{C})$ & 1440 & 9838 & -827 & 55 \\
\hline
\end{tabular}




\section{Theoretical Studies to Search Spin-Crossing and Coordinates of Calculated}

\section{Structures}

All DFT calculations were performed with the Gaussian 03 program. ${ }^{37}$

1. Estimation of the spin state inversion energy

To locate the Minimum Energy Crossing Point in the in the reaction of $\mathrm{O}_{2}$ with $\mathrm{Zr}\left(\mathrm{NH}_{2}\right)_{4}$ (5), we employed the method of partial optimization. ${ }^{\text {S1 }}$ At first, we chose the O-O bond length as the reaction coordinate. A set of partial geometry optimizations were performed on each spin state while holding the value of the chosen coordinate fixed. The results are listed in Table S11 and plotted in Figure S3.

From Figure S3, a crossing point of two spin states was found at O-O bond length of 1.30 $\AA ̊$ in the energy diagram, with an energy of ca. -420.87 Hatree. But in different state, the geometries with O-O bond length of $1.30 \AA$ are quite different. Since the Franck-Condon principle requires that both energies and geometries be the same in order for the spin hopping to take place, we realized that this is not the true MECP because that the O-O bond length is not right single geometrical coordinate in the two spin states. Later, we chose Zr-O bond length and Zr-N bond length as the reaction coordinates. The results are listed in the Tables S12 and S13, and plotted in the Figures S4 and S5, respectively. The same problem remained. The geometries of two states are very different at the "crossing point." 
Table S11. Results of calculations using the O-O bond length as the reaction coordinate

\begin{tabular}{|c|c|c|}
\hline $\begin{array}{c}\text { O-O bond } \\
\text { length }(\AA)\end{array}$ & $\begin{array}{c}\text { Energy } \\
\text { (Hartree) }\end{array}$ & $\begin{array}{c}\text { Energy } \\
\text { (Hartree) }\end{array}$ \\
\hline 1.20 & -420.877751882 & -420.834457084 \\
\hline 1.25 & -420.876360439 & -420.859028312 \\
\hline 1.30 & -420.869385498 & -420.871776021 \\
\hline 1.35 & -420.856679967 & -420.879438221 \\
\hline 1.40 & -420.847640429 & -420.883069411 \\
\hline 1.45 & -420.838222401 & -420.884255045 \\
\hline 1.50 & -420.828581400 & -420.883827009 \\
\hline
\end{tabular}

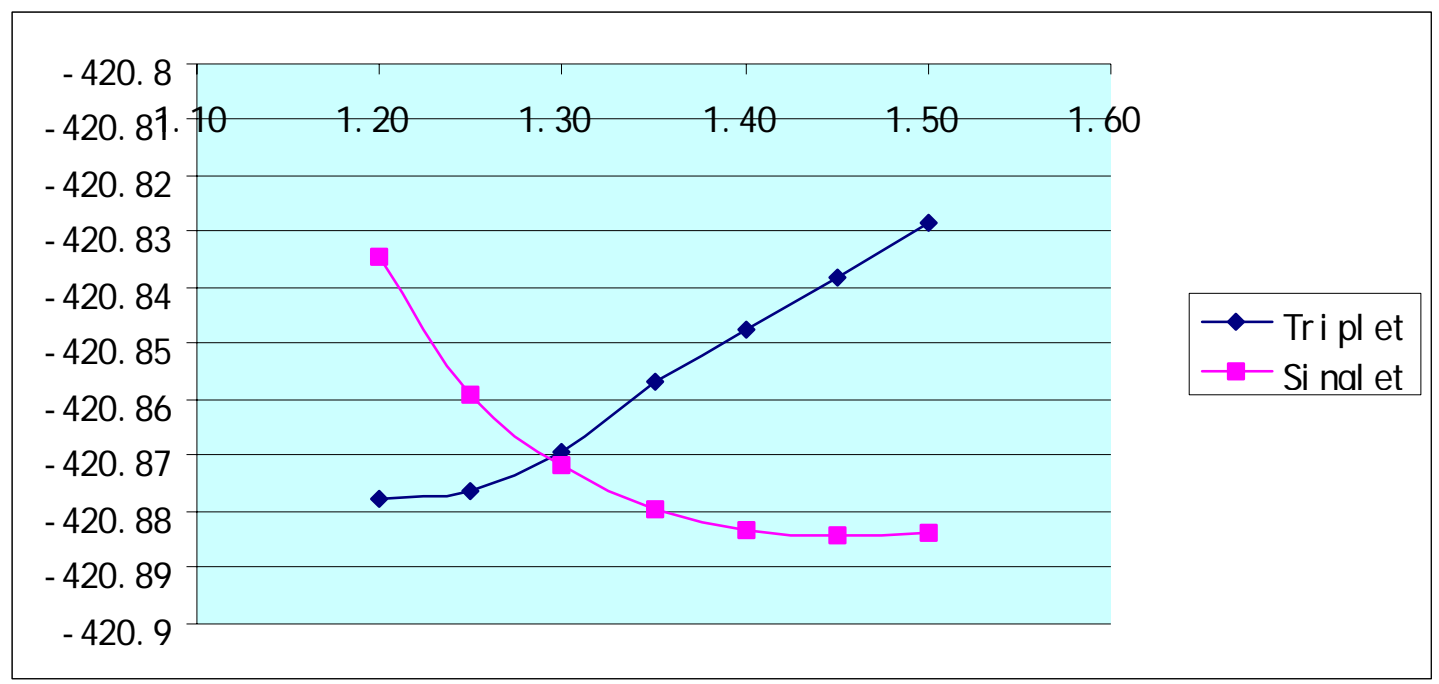

Figure S3. Results of calculations using the O-O bond length as the reaction coordinate. 
Table S12. Results of calculations using the Zr-O bond length as the reaction coordinate

\begin{tabular}{|c|c|c|}
\hline $\begin{array}{c}\text { Zr-O bond } \\
\text { length }(\AA)\end{array}$ & $\begin{array}{c}\text { Energy } \\
\text { (Hartree) }\end{array}$ & $\begin{array}{c}\text { Energy } \\
\text { (Hartree) }\end{array}$ \\
\hline 1.9 & & -420.867220782 \\
\hline 2 & & -420.875097419 \\
\hline 2.2 & -420.855184290 & -420.874602218 \\
\hline 2.4 & -420.868413731 & -420.869054474 \\
\hline 2.6 & -420.870029656 & -420.853602785 \\
\hline 2.8 & -420.872209749 & -420.839157096 \\
\hline 3 & -420.874180985 & -420.824181316 \\
\hline 3.5 & -420.875352798 & \\
\hline
\end{tabular}

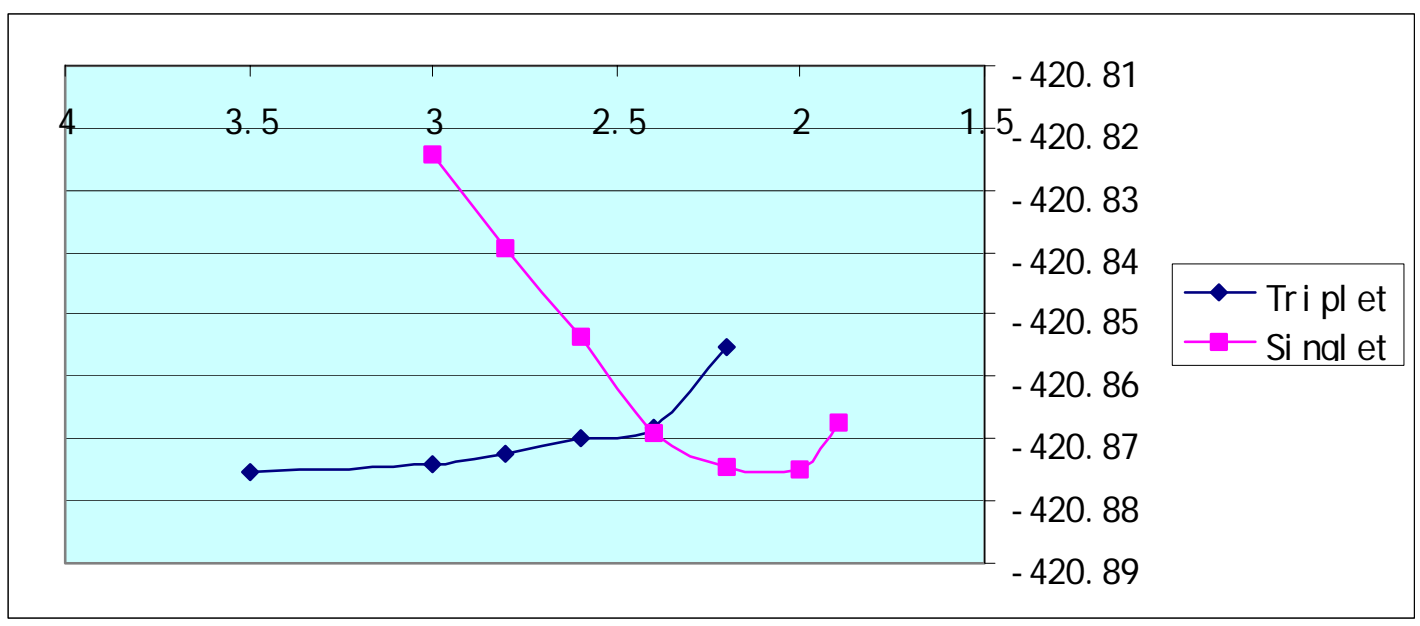

Figure S4. Results of calculations using the Zr-O bond length as the reaction coordinate. 
Table S13. Results of calculations using the $\mathrm{Zr}-\mathrm{N}$ bond length as the reaction coordinate.

\begin{tabular}{|c|c|c|}
\hline $\begin{array}{c}\text { Zr-N bond } \\
\text { length }(\AA)\end{array}$ & $\begin{array}{c}\text { Energy } \\
\text { (Hartree) }\end{array}$ & $\begin{array}{c}\text { Energy } \\
\text { (Hartree) }\end{array}$ \\
\hline 2.00 & -420.876588345 & -420.843688505 \\
\hline 2.10 & -420.877595355 & -420.862062386 \\
\hline 2.15 & -420.876035158 & -420.868643666 \\
\hline 2.20 & -420.856673769 & -420.873747728 \\
\hline 2.30 & -420.855494128 & -420.880431159 \\
\hline 2.40 & -420.852688753 & -420.883706457 \\
\hline 2.50 & -420.849079410 & -420.884770941 \\
\hline
\end{tabular}

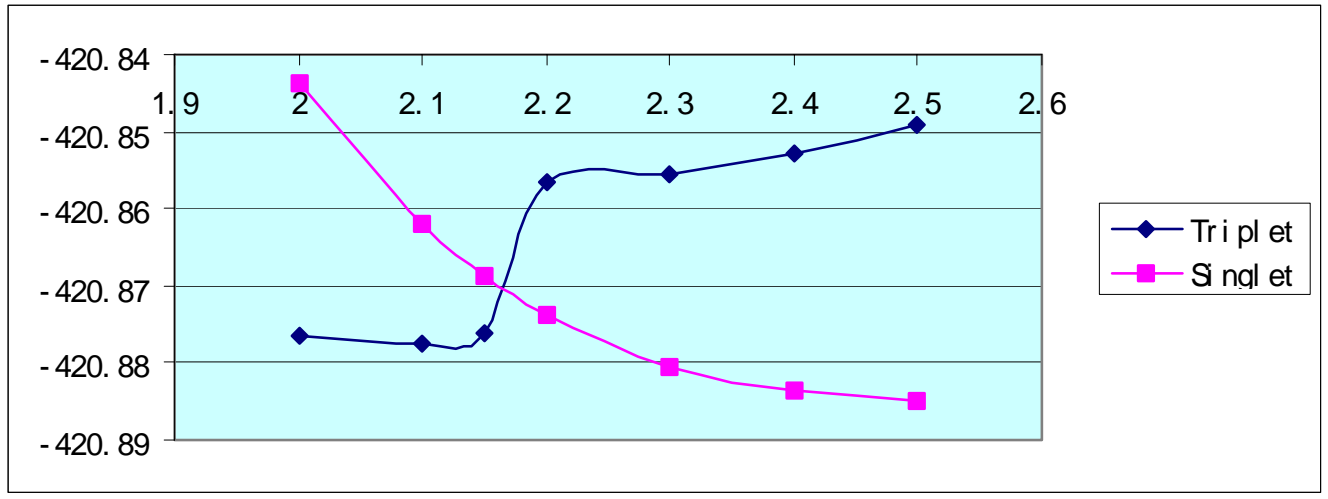

Figure S5. Results of calculations using the Zr-N bond length as the reaction coordinate. 
No real crossing point was located in the three attempts, indicating that the right reaction coordinate is not the simple bond lengths. Although we could not find the two-state crossing point, three plotted figures revealed an interesting picture - The energies of the three pseudocrossing points are very close. That means the energy at the pseudo-crossing points should be close to the real crossing point in the 3N-6 dimensional diagram. We therefore took the energy of -420.870 Hartree to estimate the spin state inversion barrier. That is the way we derived the barrier of $6.2 \mathrm{kcal} / \mathrm{mol}$ in Scheme 1 . 
Table S14. Calculated total energies, energies with zero-point energy corrections, and free energies of the structures in the text.

\begin{tabular}{|c|c|c|c|}
\hline & E (Hartree) & E (with ZPE) (Hartree) & Free energies (Hartree) \\
\hline $\mathbf{O}_{2}$ & -150.327577 & -150.323838 & -150.343818 \\
\hline 1 (BSI) & -584.9943953 & -584.6686703 & -584.7209963 \\
\hline (BSII) & -584.9941340 & & \\
\hline 1a (BSI) & -1166.524409 & -1165.865928 & -1165.938427 \\
\hline (BSII) & -1170.0079892 & & \\
\hline 6 (BSI) & -1316.856581 & -1316.191336 & -1316.265841 \\
\hline (BSII) & -1320.3396981 & & \\
\hline 5 & -270.552350 & -270.454526 & -270.489359 \\
\hline 7 & -420.8848026 & -420.776532 & -420.813646 \\
\hline 8-TS & -420.8509873 & -420.743393 & -420.779212 \\
\hline 8-TS-Ti & -432.3325937 & -432.2231957 & -432.2585957 \\
\hline 8-TS-Hf & -423.2019153 & -423.0942733 & -423.1307473 \\
\hline 9 & -420.9476579 & -420.835555 & -420.870943 \\
\hline 10-radical & -364.9649245 & -364.882557 & -364.871754 \\
\hline •NH2 & -55.8788678 & -55.859866 & -55.878192 \\
\hline 10 & -691.5230471 & -691.309930 & -691.361316 \\
\hline 10-TS-iso & -691.5170522 & -691.304800 & -691.281870 \\
\hline 11 & -691.5278589 & -691.315631 & -691.366184 \\
\hline 12 & -635.6044638 & -635.421865 & -635.470570 \\
\hline$\left(\mathrm{NH}_{2}\right)_{2}$ & -111.8690118 & -111.815387 & -111.837325 \\
\hline 13'-peroxo & -579.6244044 & -579.472695 & -579.520172 \\
\hline 13'-diradical & -579.6221887 & -579.463911 & -579.508307 \\
\hline 13 & -579.6690039 & -579.508496 & -579.552934 \\
\hline 14 & -850.3199884 & -850.057273 & -850.111909 \\
\hline 15 & -691.4612666 & -691.253783 & -691.304047 \\
\hline 16 & -691.4693182 & -691.257592 & -691.305284 \\
\hline 17-TS & -691.4555221 & -691.244323 & -691.291295 \\
\hline
\end{tabular}




\section{The structures with Cartesian coordinates.}

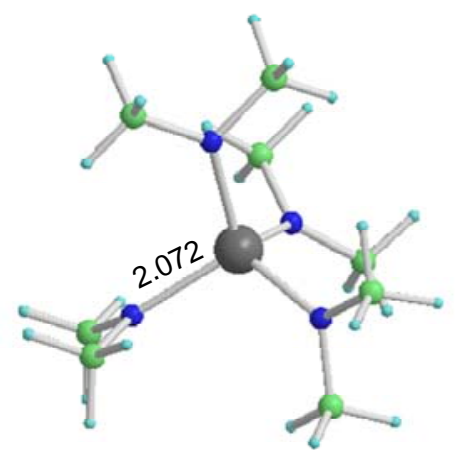

$1 E=-582.9325985$ Hatree

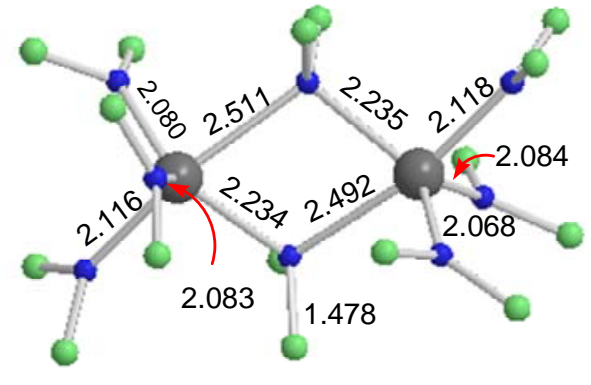

1a $E=-1165.865928$ Hatree

$\begin{array}{lrrr}\mathrm{C} & 0.000000 & 0.000000 & 0.000000 \\ \mathrm{~N} & 0.000000 & 1.463689 & 0.000000 \\ \mathrm{C} & 1.358513 & 1.995593 & 0.000000 \\ \mathrm{Zr} & -1.809484 & 2.465723 & 0.088680 \\ \mathrm{~N} & -2.976668 & 1.671766 & 1.609149 \\ \mathrm{C} & -4.400700 & 1.353221 & 1.568826 \\ \mathrm{~N} & -1.428251 & 4.460746 & 0.498851 \\ \mathrm{C} & -0.992535 & 5.370524 & -0.561014 \\ \mathrm{~N} & -2.788810 & 2.298717 & -1.730976 \\ \mathrm{C} & -3.958919 & 3.136769 & -1.994241 \\ \mathrm{C} & -1.611975 & 5.170012 & 1.761580 \\ \mathrm{C} & -2.449790 & 1.478737 & -2.889148 \\ \mathrm{C} & -2.383067 & 1.282441 & 2.888102 \\ \mathrm{H} & 0.484890 & -0.404404 & -0.905749 \\ \mathrm{H} & 0.535853 & -0.406055 & 0.876055 \\ \mathrm{H} & -1.027536 & -0.390954 & 0.030518 \\ \mathrm{H} & 1.918924 & 1.669750 & -0.894176 \\ \mathrm{H} & 1.335020 & 3.090720 & 0.009851 \\ \mathrm{H} & 1.926191 & 1.657119 & 0.885395 \\ \mathrm{H} & -4.956360 & 1.890521 & 2.357712 \\ \mathrm{H} & -4.827638 & 1.634771 & 0.599178 \\ \mathrm{H} & -4.579487 & 0.273025 & 1.716359 \\ \mathrm{H} & -0.017515 & 5.829803 & -0.320791 \\ \mathrm{H} & -0.884886 & 4.833045 & -1.513146 \\ \mathrm{H} & -1.717325 & 6.188917 & -0.717608 \\ \mathrm{H} & -4.856411 & 2.526952 & -2.199286 \\ \mathrm{H} & -4.182071 & 3.778489 & -1.129334 \\ \mathrm{H} & -3.797322 & 3.797751 & -2.863965 \\ \mathrm{H} & -0.671206 & 5.639013 & 2.100379 \\ \mathrm{H} & -2.366080 & 5.972287 & 1.670518 \\ \mathrm{H} & -1.948838 & 4.477429 & 2.541103 \\ \mathrm{H} & -3.279118 & 0.802362 & -3.162276 \\ \mathrm{H} & -2.226351 & 2.100286 & -3.774794 \\ \mathrm{H} & -1.566201 & 0.867295 & -2.673761 \\ \mathrm{H} & -2.838698 & 1.834159 & 3.729276 \\ \mathrm{H} & -2.516268 & 0.204048 & 3.085875 \\ \mathrm{H} & -1.304375 & 1.491166 & 2.894168\end{array}$

Figure S6a.

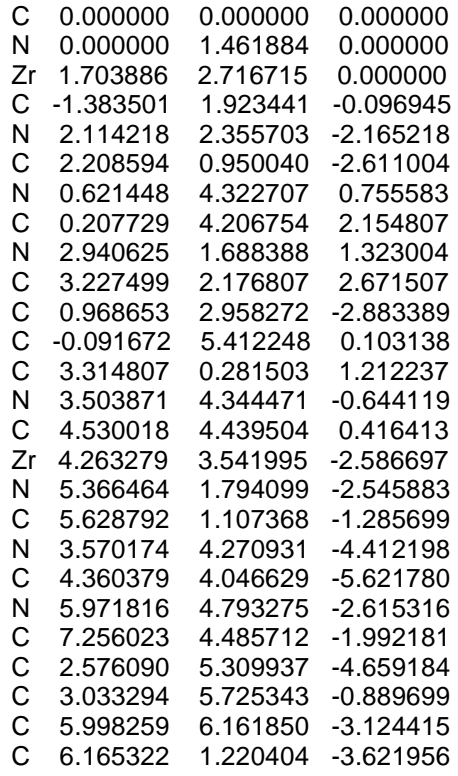

$\begin{array}{llll}\text { H } & -0.574272 & -0.397013 & 0.857804\end{array}$

H $-0.463973 \quad-0.414033 \quad-0.915432$

H $1.015341 \quad-0.401611 \quad 0.070253$

H $\quad-1.859180 \quad 1.603885-1.044330$

$\begin{array}{llll}H & -2.000704 & 1.510179 & 0.722918\end{array}$

$\begin{array}{llll}H & -1.430787 & 3.013903 & -0.039638\end{array}$

H $2.990753 \quad 0.419108 \quad-2.059367$

H $1.250874 \quad 0.423354 \quad-2.470604$

H $2.471704 \quad 0.896137 \quad-3.681020$

$\begin{array}{llll}\mathrm{H} & 0.489727 & 5.102120 & 2.736844\end{array}$

$\mathrm{H}-0.886116 \quad 4.077046 \quad 2.243043$

H $0.674118 \quad 3.3363652 .634450$

H $2.667478 \quad 1.612405 \quad 3.439293$

H $4.302024 \quad 2.080496 \quad 2.908749$

$\begin{array}{llll}\text { H } & 2.966703 & 3.236939 & 2.771837\end{array}$

H $1.172059 \quad 3.012571 \quad-3.962829$

H $0.046631 \quad 2.375162 \quad-2.724221$

$\begin{array}{llll}H & 0.780887 & 3.985428 & -2.537921\end{array}$

$\begin{array}{llll}\mathrm{H} & -1.187634 & 5.281384 & 0.180522\end{array}$

$\begin{array}{llll}\text { H } & 0.149939 & 6.391928 & 0.552405\end{array}$

H $0.159291 \quad 5.463394 \quad-0.962212$

$\begin{array}{llll}\mathrm{H} & 2.733394 & -0.354761 & 1.903008\end{array}$

H $3.152360 \quad-0.0898950 .193986$

H $4.383723 \quad 0.134565 \quad 1.447776$

$\begin{array}{llll}\mathrm{H} & 5.330559 & 5.140835 & 0.132338\end{array}$

H $4.081606 \quad 4.800222 \quad 1.358388$

H $4.975442 \quad 3.460270 \quad 0.615556$

H $\quad 6.697845 \quad 1.162226 \quad-1.006387$

H $\quad 5.045240 \quad 1.547160 \quad-0.467101$

$\begin{array}{llll}\text { H } & 5.359892 & 0.036941 & -1.342727\end{array}$

H $3.726023 \quad 3.698771 \quad-6.456019$

H $4.873668 \quad 4.968780 \quad-5.949844$

H $\quad 5.130166 \quad 3.286319-5.451464$

H $\quad 8.090074 \quad 4.630840 \quad-2.704916$

H $7.4675615 .135128-1.120191$

H $\quad 7.279094 \quad 3.444068$-1.655614

H $\quad 1.870837 \quad 5.015920 \quad-5.456759$

H $\quad 1.987213 \quad 5.518004-3.759686$

H $3.049324 \quad 6.258548$-4.976080

H $2.285131 \quad 5.755482 \quad-1.691659$

$\begin{array}{llll}H & 2.558297 & 6.136281 & 0.013367\end{array}$

$\mathrm{H} \quad 3.866224 \quad 6.390916 \quad-1.173030$

H $\quad 6.123996 \quad 6.907673 \quad-2.314997$

H $\quad 6.839776 \quad 6.311933 \quad-3.826966$

H $5.070291 \quad 6.391527 \quad-3.659610$

H $7.247028 \quad 1.294041-3.407628$

$\begin{array}{llll}\mathrm{H} & 5.927312 & 0.152457 & -3.772760\end{array}$

H $\quad 5.977089 \quad 1.740677 \quad-4.566521$ 


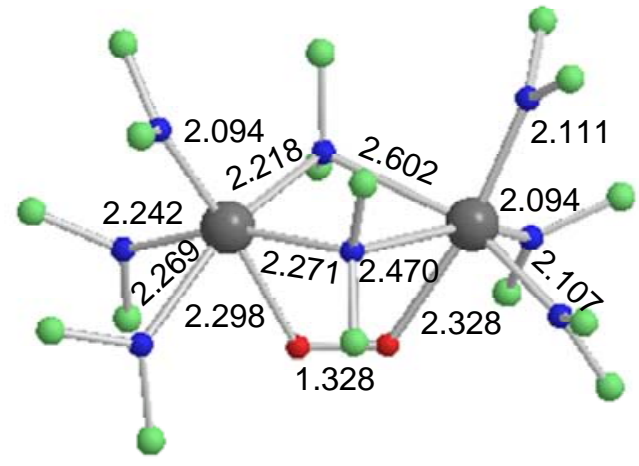

Zr-Zr distance: $3.611 \AA$

$6 \mathrm{E}=-1316.191336$ Hartree

$\begin{array}{lrrr}\mathrm{N} & -2.70948100 & 1.86981300 & -0.57283200 \\ \mathrm{Zr} & -1.68563700 & 0.11631200 & -0.06155400 \\ \mathrm{~N} & -0.00496600 & 0.97584500 & 1.20308300 \\ \mathrm{Zr} & 1.92173000 & -0.02434000 & 0.02892400 \\ \mathrm{~N} & 3.04832700 & -0.07610900 & 1.80850100 \\ \mathrm{~N} & -3.11853900 & -0.45630200 & 1.60256800 \\ \mathrm{~N} & -0.10937900 & -0.16695100 & -1.59449600 \\ \mathrm{~N} & -3.08851500 & -1.39324300 & -0.94304100 \\ \mathrm{~N} & 2.93717500 & -1.60343000 & -0.89546700 \\ \mathrm{~N} & 2.78632400 & 1.62552700 & -0.96427800 \\ \mathrm{O} & -0.66740700 & -1.76331900 & 0.77873500 \\ \mathrm{O} & 0.65913700 & -1.83002900 & 0.78358000 \\ \mathrm{C} & 3.26412000 & 0.98194600 & 2.78820100 \\ \mathrm{C} & 3.30521700 & 2.83761900 & -0.33672600 \\ \mathrm{C} & 3.14522100 & 1.64123400 & -2.38074800 \\ \mathrm{C} & 2.64907800 & -3.02773800 & -1.02376400 \\ \mathrm{C} & 4.30484300 & -1.34744200 & -1.36524700 \\ \mathrm{C} & -0.07511800 & -1.43906300 & -2.34376800 \\ \mathrm{C} & -0.10390700 & 0.62602100 & 2.64170000 \\ \mathrm{C} & -2.98965900 & -2.83878700 & -0.79118800 \\ \mathrm{C} & -3.01912500 & -1.61368800 & 2.48381000 \\ \mathrm{C} & -4.47263200 & 0.08231600 & 1.66643900 \\ \mathrm{C} & -4.39915800 & -1.03893900 & -1.47270400 \\ \mathrm{C} & -3.11358500 & 2.23488800 & -1.93159000 \\ \mathrm{C} & 0.11758500 & 2.45124000 & 1.11532100 \\ \mathrm{C} & -0.08536400 & 0.92318800 & -2.59573300 \\ \mathrm{C} & -3.11388800 & 2.92828700 & 0.35228400 \\ \mathrm{C} & 3.76957100 & -1.27325300 & 2.24494500 \\ & & & \end{array}$

Figure S6b.

$\begin{array}{rrrr}\mathrm{H} & 2.94758500 & 0.67667100 & 3.80271500 \\ \mathrm{H} & 4.33574400 & 1.24763700 & 2.85090400 \\ \mathrm{H} & 2.71206200 & 1.89054000 & 2.52855100 \\ \mathrm{H} & 3.66604000 & -2.07147000 & 1.50772800 \\ \mathrm{H} & 4.84614900 & -1.06049800 & 2.38204400 \\ \mathrm{H} & 3.38351800 & -1.64113200 & 3.21318100 \\ \mathrm{H} & 4.40204100 & 2.91374500 & -0.45577300 \\ \mathrm{H} & 2.86469100 & 3.74972500 & -0.78228600 \\ \mathrm{H} & 3.09157500 & 2.85494300 & 0.73518900 \\ \mathrm{H} & 2.65234600 & 2.46732600 & -2.92561800 \\ \mathrm{H} & 4.23591200 & 1.77498000 & -2.50915000 \\ \mathrm{H} & 2.87218100 & 0.69907300 & -2.86743500 \\ \mathrm{H} & 2.65908200 & -3.34923600 & -2.08134300 \\ \mathrm{H} & 3.40737400 & -3.63663100 & -0.49580400 \\ \mathrm{H} & 1.67353000 & -3.26180700 & -0.59383600 \\ \mathrm{H} & 5.03408900 & -1.94043900 & -0.78347500 \\ \mathrm{H} & 4.42508000 & -1.62885200 & -2.42717600 \\ \mathrm{H} & 4.56890100 & -0.29210300 & -1.25605300 \\ \mathrm{H} & -0.94232100 & -1.52037400 & -3.02334500 \\ \mathrm{H} & 0.84198200 & -1.51436500 & -2.94867100 \\ \mathrm{H} & -0.09567000 & -2.28850600 & -1.65961400 \\ \mathrm{H} & 0.74877800 & 1.02117000 & 3.20831400 \\ \mathrm{H} & -1.02370500 & 1.04482200 & 3.08662400 \\ \mathrm{H} & -0.11981600 & -0.45742500 & 2.76892500 \\ \mathrm{H} & -3.77906000 & -3.22059300 & -0.11730300 \\ \mathrm{H} & -3.12645700 & -3.33824500 & -1.76565600 \\ \mathrm{H} & -2.01927400 & -3.11626000 & -0.37709700 \\ \mathrm{H} & -0.94423000 & 0.85599000 & -3.28670400 \\ \mathrm{H} & -0.09773400 & 1.90728300 & -2.11593800 \\ \mathrm{H} & 0.81974200 & 0.86565200 & -3.21460600 \\ \mathrm{H} & -2.59706800 & 3.87764200 & 0.13118400 \\ \mathrm{H} & -4.19952600 & 3.12372000 & 0.28373300 \\ \mathrm{H} & -2.88866100 & 2.65400300 & 1.38865500 \\ \mathrm{H} & 1.03886500 & 2.80448700 & 1.60619300 \\ \mathrm{H} & 0.14384300 & 2.78341900 & 0.07276700 \\ \mathrm{H} & -0.71906300 & 2.95823500 & 1.62126400 \\ \mathrm{H} & -4.20057600 & 2.42926300 & -1.98353700 \\ \mathrm{H} & -2.59978700 & 3.14914300 & -2.27608600 \\ \mathrm{H} & -2.88371900 & 1.43544800 & -2.64410200 \\ \mathrm{H} & -4.55460900 & -1.50882700 & -2.45949200 \\ \mathrm{H} & -5.20951600 & -1.40082700 & -0.81295700 \\ \mathrm{H} & -4.49996300 & 0.04285900 & -1.57832000 \\ \mathrm{H} & -5.21997600 & -0.68087500 & 1.38030900 \\ \mathrm{H} & -4.71143400 & 0.39302900 & 2.69828600 \\ \mathrm{H} & -4.58297200 & 0.94001800 & 1.00291700 \\ \mathrm{H} & -3.26839400 & -1.32287900 & 3.51870900 \\ \mathrm{H} & -3.73705100 & -2.40106200 & 2.18729900 \\ \mathrm{H} & -2.01395500 & -2.03357100 & 2.45687900\end{array}$




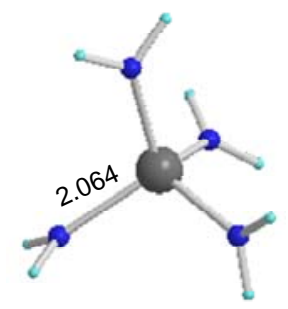

$5 E=-270.454526$ Hatree
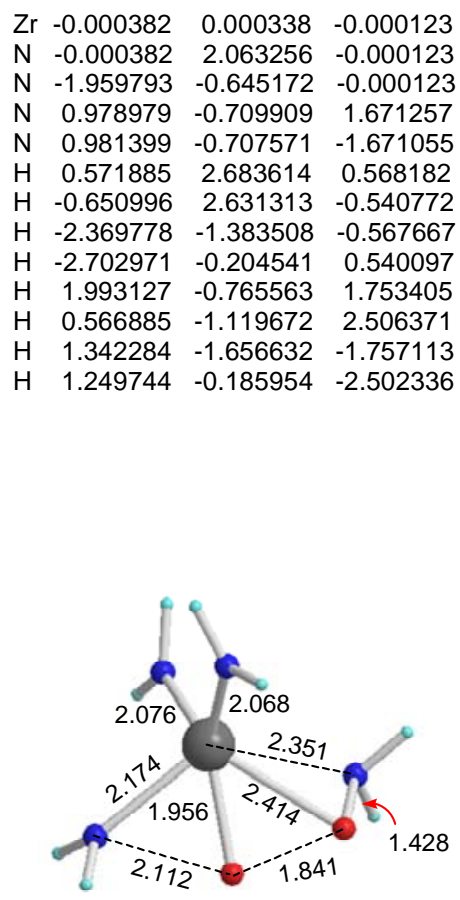

8-TS $E=-420.743393$ Hatree

$\begin{array}{lrrr}\mathrm{N} & -0.235599 & -2.072088 & -0.083815 \\ \mathrm{Zr} & -0.235599 & 0.003280 & -0.083815 \\ \mathrm{~N} & 2.083912 & 0.389103 & -0.083815 \\ \mathrm{O} & 1.496145 & 1.670513 & 0.135780 \\ \mathrm{O} & 0.257981 & 1.171663 & 1.404158 \\ \mathrm{~N} & -0.863831 & 0.766935 & -1.898545 \\ \mathrm{~N} & -1.597509 & 0.182047 & 1.601690 \\ \mathrm{H} & -0.265894 & -2.637453 & -0.932191 \\ \mathrm{H} & -0.136444 & -2.709818 & 0.703940 \\ \mathrm{H} & 2.609597 & 0.454266 & -0.958645 \\ \mathrm{H} & 2.736445 & 0.196645 & 0.680264 \\ \mathrm{H} & -1.458583 & 0.295400 & -2.578847 \\ \mathrm{H} & -0.749054 & 1.735518 & -2.192992 \\ \mathrm{H} & -1.954776 & 1.069823 & 1.946874 \\ \mathrm{H} & -1.686793 & -0.515834 & 2.339960\end{array}$

Figure S6c.

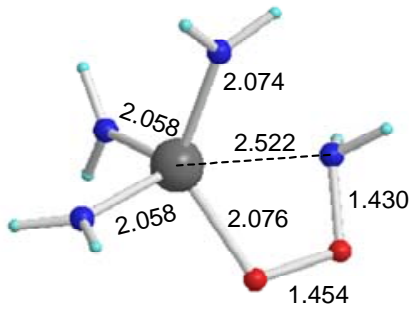

$7 \quad E=-420.776532$ Hatree
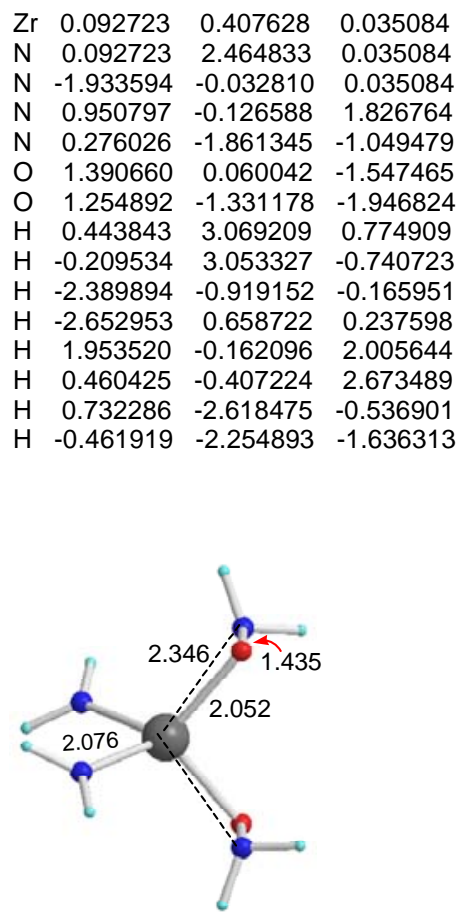

$9\left(C_{2}\right) \quad E=-420.835555$ Hatree

$\begin{array}{lrrr}\mathrm{Zr} & -0.073771 & 0.069220 & -0.001625 \\ \mathrm{~N} & -0.073771 & 2.144830 & -0.001625 \\ \mathrm{~N} & -2.142610 & -0.095127 & -0.001625 \\ \mathrm{O} & 0.783886 & -0.868507 & 1.609656 \\ \mathrm{O} & 0.922847 & -0.711119 & -1.616737 \\ \mathrm{~N} & -0.024986 & -1.775399 & -1.451108 \\ \mathrm{~N} & 1.763337 & 0.168120 & 1.453842 \\ \mathrm{H} & 0.674644 & 2.748701 & 0.329917 \\ \mathrm{H} & -0.780741 & 2.726144 & -0.448277 \\ \mathrm{H} & -2.684960 & -0.894162 & -0.321282 \\ \mathrm{H} & -2.777721 & 0.566746 & 0.440865 \\ \mathrm{H} & -0.554294 & -1.863290 & -2.319045 \\ \mathrm{H} & 0.494778 & -2.640502 & -1.297188 \\ \mathrm{H} & 1.802784 & 0.698185 & 2.324919 \\ \mathrm{H} & 2.670577 & -0.273840 & 1.299313\end{array}$




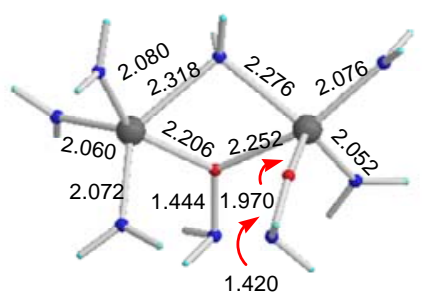

$10 \mathrm{E}=-691.30993$ Hatree

$\begin{array}{llll}N & -1.567854 & -2.815776 & 0.341960\end{array}$

Zr $-1.567854 \quad-0.736104 \quad 0.341960$

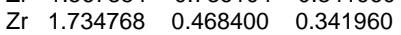

$\begin{array}{lllll}\mathrm{N} & 2.090259 & 2.487341 & 0.391038\end{array}$

$\begin{array}{llll}\mathrm{N} & -3.215984 & -0.271226 & 1.487173\end{array}$

$\begin{array}{llll}N & -1.967277 & -0.346446 & -1.653099\end{array}$

$\begin{array}{llll}N & 0.372324 & -0.846738 & 1.604467\end{array}$

$\begin{array}{llll}\mathrm{O} & -0.412757 & 1.142861 & 0.394973\end{array}$

$\begin{array}{lllll}N & -0.840406 & 2.331566 & -0.301943\end{array}$

$\begin{array}{llll}\mathrm{O} & 1.552671 & -0.406235 & -1.412440\end{array}$

$\begin{array}{llll}\mathrm{N} & 1.112848 & -0.302410 & -2.758173\end{array}$

$\begin{array}{llll}N & 3.573173 & -0.170448 & 1.064579\end{array}$

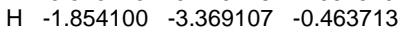

H $\quad-1.170703 \quad-3.447936 \quad 1.034009$

$\begin{array}{llll}H & 2.978464 & 2.974796 & 0.472470\end{array}$

H $1.326308 \quad 3.1423810 .221156$

$\begin{array}{llll}\text { H } & -3.508115 & 0.663429 & 1.767627\end{array}$

H $-3.869121 \quad-0.947425 \quad 1.875329$

H $-1.218856-0.314714 \quad-2.351908$

$\begin{array}{llll}\text { H } & -2.872508 & -0.293381 & -2.114858\end{array}$

H $\quad 0.164722 \quad-0.420014 \quad 2.511262$

H $0.756184-1.769752 \quad 1.808471$

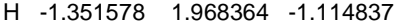

$\begin{array}{llll}\text { H } & -1.546439 & 2.743906 & 0.312464\end{array}$

$\begin{array}{llll}H & 1.658951 & 0.460944 & -3.166382\end{array}$

$\begin{array}{llll}\text { H } & 1.455438 & -1.159438 & -3.198797\end{array}$

$\begin{array}{llll}\mathrm{H} & 4.050516 & 0.150194 & 1.905837\end{array}$

$\begin{array}{llll}\text { H } & 4.136923 & -0.917032 & 0.659414\end{array}$

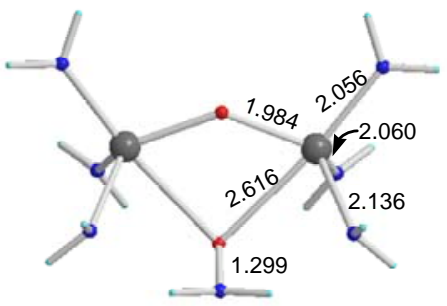

$12\left(C_{2}\right) \mathrm{E}=-635.421865$ Hatree

N $0.639289-3.509182 \quad-1.002118$

Zr $0.639289-1.453122 \quad-1.002118$

N $2.698341-0.886806 \quad-1.002118$

$\begin{array}{llll}\text { O } & -0.383690 & -0.969434 & 0.627412\end{array}$

$\begin{array}{lll}\mathrm{Zr}-1.187327 & 0.729334 & 1.260411\end{array}$

$\begin{array}{llll}\mathrm{N} & 0.178630 & 2.127305 & 2.121898\end{array}$

N $-0.150229 \quad-0.961873 \quad-2.840310$

$\begin{array}{llll}\mathrm{O} & 0.477647 & 1.135487 & -0.714102\end{array}$

$\begin{array}{llll}\text { N } & 1.598377 & 1.646811 & -0.300654\end{array}$

N $-2.426528 \quad 1.767916 \quad-0.016172$

$\begin{array}{lll}\text { N }-2.431788 & 0.157762 & 2.793955\end{array}$

H $-0.000957 \quad-4.091016-0.464014$

H $1.294044 \quad-4.119110-1.487888$

H $3.198664-0.800441-1.887235$

H $3.354362 \quad-1.279028-0.326022$

H $0.692933 \quad 1.905773 \quad 2.974996$

H $-0.077040 \quad 3.113686 \quad 2.176113$

H $-0.416565-0.013460-3.098165$

H $-0.472151 \quad-1.605118$-3.559324

H $1.483459 \quad 2.125149 \quad 0.618069$

H $2.405355 \quad 1.025660 \quad-0.520984$

H $-3.423672 \quad 1.932046 \quad 0.098603$

H $-2.144520 \quad 2.067410 \quad-0.947664$

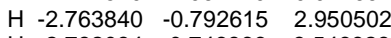

$\begin{array}{lll}\mathrm{H}-2.782084 & 0.746866 & 3.546928\end{array}$

Figure S6d.

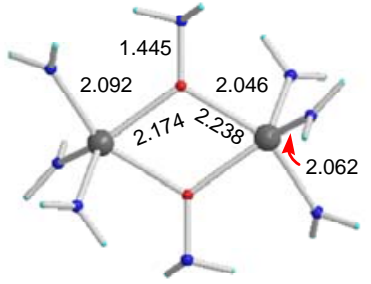

$11\left(C_{2}\right) \mathrm{E}=-691.315631$ Hatree

$\begin{array}{llll}N & 1.010633 & -2.618783 & 1.427539\end{array}$

$\begin{array}{llll}\mathrm{Zr} & 1.010633 & -0.572511 & 1.427539\end{array}$

$\begin{array}{llll}\mathrm{N} & 2.965379 & 0.080679 & 1.427539\end{array}$

$\begin{array}{llll}\text { O } & -0.587005 & 0.743010 & 0.763496\end{array}$

$\begin{array}{llll}Z r & -1.009903 & 0.574371 & -1.428899\end{array}$

$\begin{array}{llll}\mathrm{N} & -0.421001 & 0.183431 & -3.397496\end{array}$

$\begin{array}{llll}N & 0.423800 & -0.173386 & 3.395121\end{array}$

$\begin{array}{llll}\mathrm{O} & 0.595306 & -0.731861 & -0.765929\end{array}$

$\begin{array}{llll}\text { N } & 1.197297 & -1.793673 & -1.538650\end{array}$

$\begin{array}{llll}\mathrm{N} & -1.188954 & 1.804664 & 1.536208\end{array}$

$\begin{array}{llll}\text { N } & -2.955660 & -0.104274 & -1.424674\end{array}$

N $\quad-1.034874 \quad 2.620591 \quad-1.427385$

H $0.969814 \quad-3.121878 \quad 0.539641$

H $\quad 0.948310 \quad-3.264292 \quad 2.209611$

$\begin{array}{llll}H & 3.275847 & 1.046908 & 1.332820\end{array}$

$\begin{array}{llll}\text { H } & 3.787548 & -0.512602 & 1.518468\end{array}$

$\begin{array}{llll}\mathrm{H} & 0.461423 & 0.435288 & -3.841277\end{array}$

H $\quad-1.064117 \quad-0.118459-4.127112$

$\begin{array}{lrrr}\mathrm{H} & 1.070478 & 0.118693 & 4.125592\end{array}$

$\begin{array}{llll}H & -0.462826 & -0.411949 & 3.837864\end{array}$

H $2.180034 \quad-1.512583 \quad-1.613420$

H $\quad 0.796502 \quad-1.653415 \quad-2.473167$

H $\quad-2.169955 \quad 1.519680 \quad 1.618469$

H $\quad-0.781769 \quad 1.669883 \quad 2.468684$

$\begin{array}{llll}\mathrm{H} & -3.251272 & -1.075244 & -1.330620\end{array}$

$\begin{array}{llll}\text { H } & -3.786928 & 0.476998 & -1.509948\end{array}$

$\begin{array}{llll}\mathrm{H} & -0.985807 & 3.266459 & -2.210148\end{array}$

H $\quad-0.992933 \quad 3.124255 \quad-0.539864$

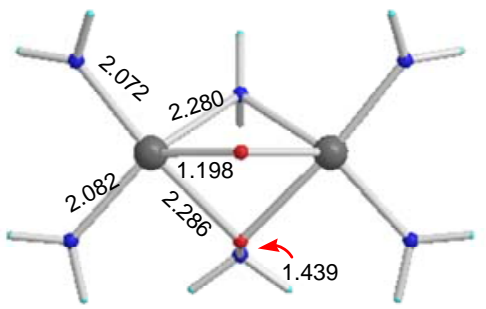

$13\left(C_{2}\right) E=-579.508496$ Hatree

$\begin{array}{llll}N & -1.010657 & -3.250874 & 0.449236\end{array}$

$\begin{array}{llll}\mathrm{Z} r & -1.010657 & -1.178486 & 0.449236\end{array}$

$\begin{array}{llll}Z r & 1.396447 & 0.678192 & 0.449236\end{array}$

$\begin{array}{llll}\mathrm{N} & 1.513051 & 2.756868 & 0.409527\end{array}$

N $\quad-3.050850 \quad-0.763648 \quad 0.408903$

N $\quad 0.532546 \quad-0.690501 \quad-1.155326$

$\begin{array}{llll}\text { O } & 0.419096 & -0.543366 & 1.658572\end{array}$

$\begin{array}{llll}\mathrm{O} & -0.828379 & 1.073813 & 0.112335\end{array}$

$\begin{array}{llll}\mathrm{N} & -1.211209 & 1.570147 & -1.183082\end{array}$

$\begin{array}{llll}\mathrm{N} & 3.401096 & 0.152666 & 0.449124\end{array}$

$\begin{array}{llll}\text { H } & -0.258676 & -3.843415 & 0.800757\end{array}$

$\begin{array}{llll}\mathrm{H} & -1.725556 & -3.868470 & 0.065371\end{array}$

$\begin{array}{llll}\mathrm{H} & 2.375980 & 3.299584 & 0.414220\end{array}$

H $\quad 0.741112 \quad 3.386830 \quad 0.618419$

$\begin{array}{llll}\mathrm{H} & -3.464575 & 0.142707 & 0.618012\end{array}$

$\begin{array}{llll}\mathrm{H} & -3.794660 & -1.460751 & 0.413020\end{array}$

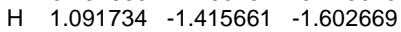

H $\quad 0.092745 \quad-0.120243 \quad-1.886168$

H $\quad-2.233939 \quad 1.555586-1.177309$

H $-0.938182 \quad 2.555860 \quad-1.176993$

$\begin{array}{llll}\text { H } & 4.179451 & 0.688012 & 0.065142\end{array}$

$\begin{array}{llll}\text { H } & 3.784082 & -0.724850 & 0.800434\end{array}$ 


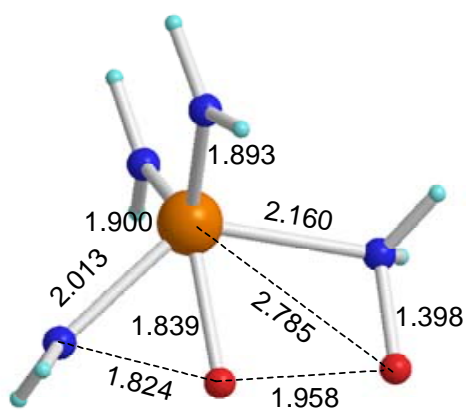

8-TS-Ti E $=-432.2231957$ Hartree

$\begin{array}{llll}N & 0.000000 & 0.000000 & 0.000000\end{array}$

$\begin{array}{llll}\mathrm{Ti} & 0.000000 & 1.899498 & 0.000000\end{array}$

$\begin{array}{llll}\mathrm{N} & 1.949941 & 2.400122 & 0.000000\end{array}$

$\begin{array}{llll}\mathrm{N} & -1.481158 & 2.188469 & -1.543641\end{array}$

$\begin{array}{llll}\text { O } & -0.866350 & 3.176537 & -2.318567\end{array}$

$\begin{array}{llll}O & 0.791257 & 2.920365 & -1.309165\end{array}$

$\begin{array}{llll}N & -0.851356 & 2.788965 & 1.438384\end{array}$

$\begin{array}{llll}\mathrm{H} & -0.306835 & -0.564956 & 0.791607\end{array}$

H $\quad 0.356612 \quad-0.620210 \quad-0.724970$

$\begin{array}{llll}H & 2.299397 & 3.309937 & 0.289343\end{array}$

H $2.672581 \quad 1.894035 \quad-0.506787$

H $\quad-2.384025 \quad 2.530444 \quad-1.199703$

H $\quad-1.669020 \quad 1.359099-2.117028$

$\begin{array}{llll}\text { H } & -1.027604 & 3.792016 & 1.474381\end{array}$

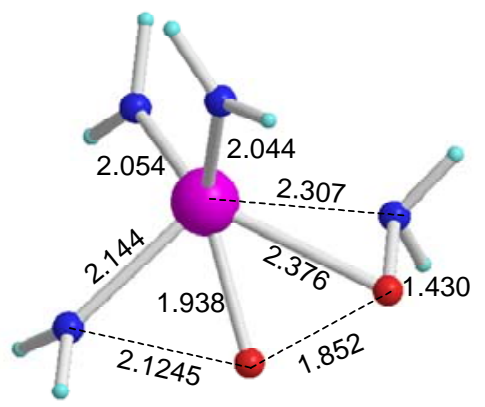

8-TS-Hf $E=-423.0942733$ Hartree

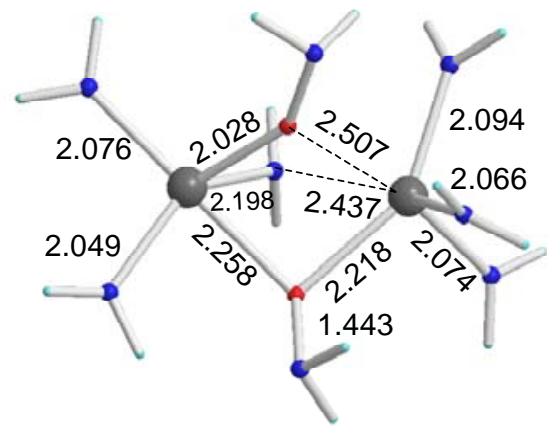

10-TS-iso $E=-691.304800$ Hartree

N $\quad 2.995656 \quad-1.369634 \quad-0.149176$

Zr $2.995656 \quad 0.724435 \quad-0.149176$

$\begin{array}{llll}\mathrm{N} & 0.560753 & 0.622719 & -0.149176\end{array}$

Zr $0.517230 \quad 1.642363 \quad-2.095504$

$\begin{array}{llll}\mathrm{N} & -0.941519 & 0.659133 & -3.197314\end{array}$

N $3.477700 \quad 1.170925 \quad 1.809960$

N $4.752267 \quad 1.185520 \quad-1.151142$

$\begin{array}{lllll}O & 1.946835 & 2.614998 & -0.645431\end{array}$

$\begin{array}{llll}\mathrm{N} & 2.644698 & 3.836434 & -0.968542\end{array}$

$\begin{array}{llll}O & 2.214293 & 0.621296 & -2.529350\end{array}$

$\begin{array}{llll}\mathrm{N} & 2.895717 & -0.276477 & -3.415604\end{array}$

$\begin{array}{llll}\mathrm{N} & 0.132153 & 3.578989 & -2.643261\end{array}$

H $3.832185 \quad-1.923876-0.326216$

$\begin{array}{llll}\text { H } & 2.241302 & -2.006525 & 0.097942\end{array}$

$\begin{array}{llll}\mathrm{H} & 0.285053 & 1.141082 & 0.685797\end{array}$

$\begin{array}{llll}\mathrm{H} & 0.138852 & -0.304517 & -0.084201\end{array}$

H $-1.705448 \quad 1.115410-3.696142$

$\begin{array}{llll}\text { H } & -1.083311 & -0.346226 & -3.292185\end{array}$

$\begin{array}{llll}\mathrm{H} & 3.497037 & 2.093190 & 2.242532\end{array}$

H $\quad 3.730410 \quad 0.483177 \quad 2.516564$

H $4.845218 \quad 1.105513 \quad-2.163797$

H $\quad 5.660255 \quad 1.395608 \quad-0.743080$

$\begin{array}{llll}H & 3552752 & 3.529587 & -1.333563\end{array}$

H $\quad 2.840427 \quad 4.257531 \quad-0.057552$

H $\quad 2.213656 \quad-0.482420-4.151010$

H $2.979203 \quad-1.131081-2.853595$

H $\quad-0.349689 \quad 3.938108 \quad-3.465025$

H $\quad 0.684438 \quad 4.323583 \quad-2.211824$

\section{Figure S6e.}
$\begin{array}{llll}N & 0.000000 & 0.000000 & 0.000000\end{array}$
$\begin{array}{llll}\text { Hf } & 0.000000 & 2.052794 & 0.000000\end{array}$
N $2.137872 \quad 2.197474 \quad 0.000000$
N $-1.510666 \quad 2.444297 \quad-1.699692$
$\begin{array}{llll}\mathrm{O} & -0.966795 & 3.727567 & -1.379099\end{array}$
$\begin{array}{llll}O & 0.816925 & 3.240700 & -1.295460\end{array}$
$\begin{array}{llll}\mathrm{N} & -0.988245 & 2.749954 & 1.646669\end{array}$
H $\quad-0.731421 \quad-0.573187 \quad 0.418700$
H $\quad 0.652779 \quad-0.630549 \quad-0.460636$
$\begin{array}{llll}\mathrm{H} & 2.642283 & 3.074783 & 0.100814\end{array}$
H $\quad 2.770930 \quad 1.500723 \quad-0.391666$
H $\quad-2.516458 \quad 2.500148 \quad-1.523892$
H $-1.357147 \quad 2.265245 \quad-2.695094$
H $-1.104923 \quad 2.251064 \quad 2.527119$
H $\quad-1.310045 \quad 3.706899 \quad 1.778939$ 


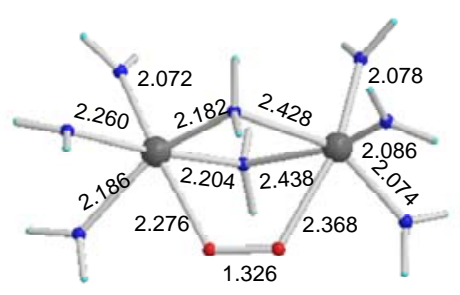

$15 E=-691.253783$ Hatree

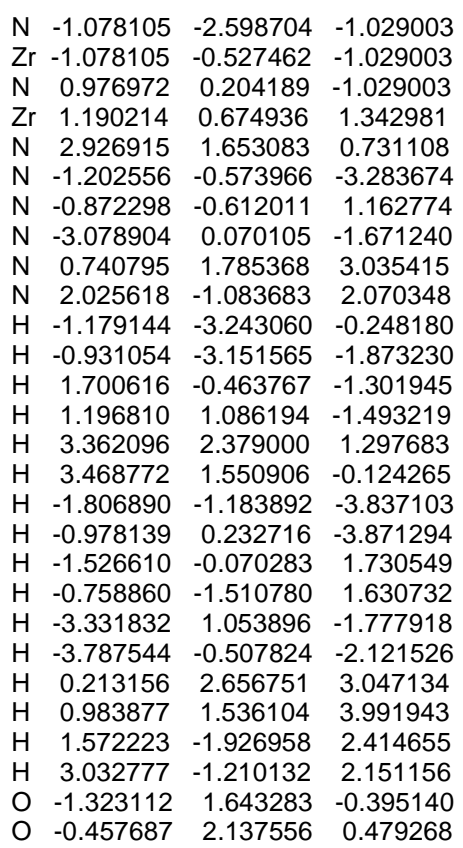

Figure S6f.

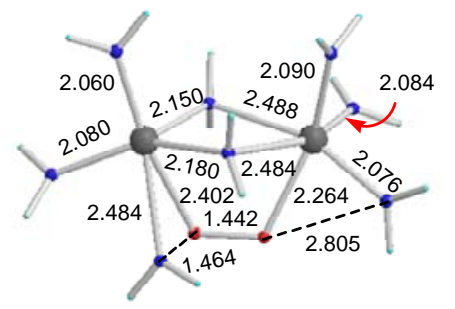

$16 \quad E=-691.257592$ Hatree

N $\quad-1.274850 \quad-2.356142 \quad-1.086998$ $\mathrm{Zr}-1.274850 \quad-0.265985-1.086998$

N $\quad \begin{array}{llll}1.191562 & 0.053026 & -1.086998\end{array}$

$\mathrm{Zr} \quad 1.428815 \quad 0.141316 \quad 1.048339$

N $\quad 2.917466 \quad 1.320130 \quad 1.894151$

O $-1.106552 \quad 1.773656 \quad-0.119153$

$\begin{array}{llll}\text { O } & 0.205309 & 2.097272 & 0.382487\end{array}$

$\begin{array}{llll}\mathrm{N} & -1.024180 & 0.010065 & -3.137563\end{array}$

N $\quad-0.592421 \quad-0.640244 \quad 1.271222$

$\begin{array}{llll}\mathrm{N} & -3.289632 & 0.189025 & -0.887402\end{array}$

$\begin{array}{llll}N & 0.127992 & 2.100235 & 1.844252\end{array}$

N $2.359592 \quad-1.682164 \quad 1.272632$

H $\quad-1.331709-3.023954-0.321521$

H $\quad-1.298467-2.876015 \quad-1.962507$

H $\quad 1.632819-0.716362 \quad-1.590576$

H $\quad 1.336065 \quad 0.901943 \quad-1.635082$

$\begin{array}{llll}\text { H } & 2.902909 & 1.986121 & 2.662724\end{array}$

H $3.878715 \quad 1.263106 \quad 1.554511$

H $-0.180443 \quad-0.048661-3.702830$

H $\quad-1.799142 \quad 0.263098 \quad-3.747525$

$\begin{array}{llll}\mathrm{H} & -1.351748 & -0.204312 & 1.791766\end{array}$

H $\quad-0.609605 \quad-1.631777 \quad 1.512329$

H $\quad-3.666903 \quad 1.121316 \quad-0.723743$

H $-4.061071 \quad-0.471861 \quad-0.948534$

$\begin{array}{llll}\mathrm{H} & 0.495651 & 3.017128 & 2.110128\end{array}$

H $\quad-0.881189 \quad 2.097856 \quad 2.016150$

H $2.075019 \quad-2.5619190 .842903$

H $\quad 3.190849-1.855896 \quad 1.833837$ 


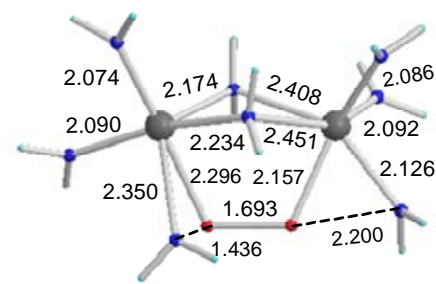

17-TS E $=-691.244323$ Hatree

$\begin{array}{lrrr}\mathrm{N} & 1.029642 & -2.759563 & 1.157521 \\ \mathrm{Zr} & 1.029642 & -0.667185 & 1.157521 \\ \mathrm{~N} & 3.115736 & -0.255617 & 1.157521 \\ \mathrm{~N} & -1.133968 & 0.386098 & 1.077773 \\ \mathrm{Zr} & -1.323850 & 0.603808 & -1.076258 \\ \mathrm{~N} & -2.836170 & -0.682842 & -1.673797 \\ \mathrm{O} & 1.741164 & 0.994116 & -0.020478 \\ \mathrm{O} & 0.495687 & 1.942908 & -0.665229 \\ \mathrm{~N} & 0.544705 & 1.629436 & -2.066190 \\ \mathrm{~N} & 0.788007 & -0.278474 & 3.192727 \\ \mathrm{~N} & 0.109884 & -1.107582 & -1.071381 \\ \mathrm{~N} & -2.417550 & 2.310060 & -1.585949 \\ \mathrm{H} & 1.081355 & -3.413714 & 0.380104 \\ \mathrm{H} & 1.164854 & -3.285628 & 2.019846 \\ \mathrm{H} & 3.517789 & 0.655953 & 1.362077 \\ \mathrm{H} & 3.801136 & -0.809814 & 0.645629 \\ \mathrm{H} & -1.847549 & -0.183366 & 1.536752 \\ \mathrm{H} & -1.036486 & 1.236358 & 1.635006 \\ \mathrm{H} & -2.757325 & -1.661005 & -1.943479 \\ \mathrm{H} & -3.816493 & -0.414185 & -1.748421 \\ \mathrm{H} & 0.520573 & 2.520209 & -2.565794 \\ \mathrm{H} & 1.460232 & 1.190039 & -2.194235 \\ \mathrm{H} & -0.047370 & -0.132019 & 3.753750 \\ \mathrm{H} & 1.597909 & -0.245693 & 3.810126 \\ \mathrm{H} & 0.895691 & -1.174657 & -1.718604 \\ \mathrm{H} & -0.304802 & -2.039730 & -1.028813 \\ \mathrm{H} & -3.097711 & 2.403004 & -2.339170 \\ \mathrm{H} & -2.274731 & 3.239087 & -1.188555\end{array}$

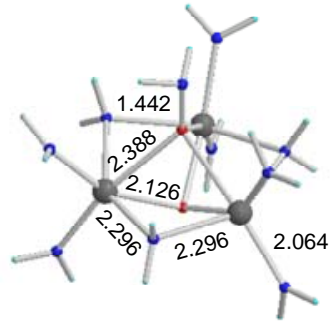

$14 E=-850.057273$ Hatree
$\begin{array}{lrrr}\mathrm{N} & -0.131898 & -3.427795 & 1.328305 \\ \mathrm{Zr} & -0.131898 & -1.364342 & 1.328305 \\ \mathrm{~N} & 2.048569 & -0.794120 & 1.328305 \\ \mathrm{Zr} & 1.837803 & 0.350720 & -0.681944 \\ \mathrm{~N} & 3.386693 & -0.371141 & -1.852600 \\ \mathrm{~N} & -0.509249 & -0.783433 & 3.314866 \\ \mathrm{~N} & -2.242533 & -0.850894 & 0.585158 \\ \mathrm{Zr} & -1.395950 & 0.167774 & -1.290084 \\ \mathrm{~N} & -2.671150 & 1.836328 & -1.160579 \\ \mathrm{O} & 0.245792 & -1.067743 & -0.743001 \\ \mathrm{O} & -0.104898 & 0.902951 & 0.578271 \\ \mathrm{~N} & -0.289951 & 2.096868 & 1.365123 \\ \mathrm{~N} & 2.451867 & 2.160405 & 0.077272 \\ \mathrm{~N} & 0.343716 & 1.270236 & -2.205037 \\ \mathrm{~N} & -2.136565 & -1.000025 & -2.821777 \\ \mathrm{H} & 0.015010 & -3.999750 & 0.497432 \\ \mathrm{H} & -0.283813 & -4.047118 & 2.122168 \\ \mathrm{H} & 2.385286 & -0.134800 & 2.030229 \\ \mathrm{H} & 2.700740 & -1.579683 & 1.326959 \\ \mathrm{H} & 4.282331 & 0.085050 & -2.024461 \\ \mathrm{H} & 3.390060 & -1.252023 & -2.366159 \\ \mathrm{H} & 0.197429 & -0.544201 & 4.009603 \\ \mathrm{H} & -1.419657 & -0.694825 & 3.764707 \\ \mathrm{H} & -2.874960 & -1.637108 & 0.430914 \\ \mathrm{H} & -2.728488 & -0.197556 & 1.201460 \\ \mathrm{H} & -3.554603 & 1.896682 & -0.655747 \\ \mathrm{H} & -2.513196 & 2.739845 & -1.605621 \\ \mathrm{H} & -1.153142 & 2.504461 & 0.992772 \\ \mathrm{H} & -0.518128 & 1.742236 & 2.298953 \\ \mathrm{H} & 1.820979 & 2.686384 & 0.688186 \\ \mathrm{H} & 3.325174 & 2.669573 & -0.047379 \\ \mathrm{H} & 0.522094 & 1.055914 & -3.187190 \\ \mathrm{H} & 0.388710 & 2.285256 & -2.113282 \\ \mathrm{H} & -2.953124 & -0.816103 & -3.401711 \\ \mathrm{H} & -1.729050 & -1.888021 & -3.112414 \\ & & & \end{array}$

\section{Figure S6g.}


Additional Reference

S1. Smith, K. M.; Poli, R.; Harvey, J. N. New J. Chem. 2000, 24, 77. 